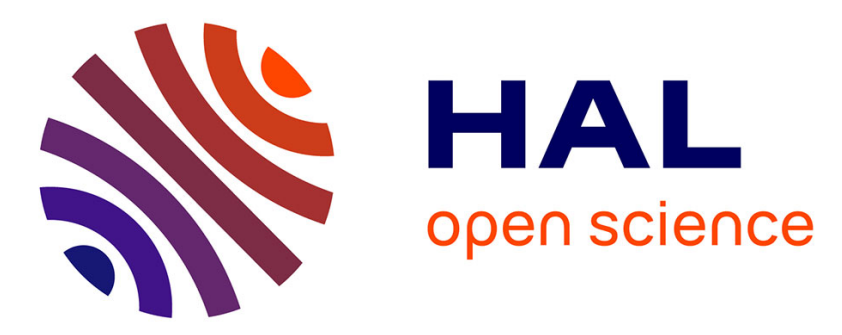

\title{
Calibrating and interpreting implicit-texture models of foam flow through porous media of different permeabilities
}

Omar Gassara, Frédéric Douarche, Benjamin Braconnier, Bernard Bourbiaux

\section{- To cite this version:}

Omar Gassara, Frédéric Douarche, Benjamin Braconnier, Bernard Bourbiaux. Calibrating and interpreting implicit-texture models of foam flow through porous media of different permeabilities. 2018. hal-01702398

\section{HAL Id: hal-01702398 \\ https://hal-ifp.archives-ouvertes.fr/hal-01702398}

Preprint submitted on 6 Feb 2018

HAL is a multi-disciplinary open access archive for the deposit and dissemination of scientific research documents, whether they are published or not. The documents may come from teaching and research institutions in France or abroad, or from public or private research centers.
L'archive ouverte pluridisciplinaire HAL, est destinée au dépôt et à la diffusion de documents scientifiques de niveau recherche, publiés ou non, émanant des établissements d'enseignement et de recherche français ou étrangers, des laboratoires publics ou privés. 


\title{
Calibrating and interpreting implicit-texture models of foam flow through porous media of different permeabilities
}

\author{
O. Gassara ${ }^{\mathrm{a}}$, F. Douarche ${ }^{\mathrm{a}, *}$, B. Braconnier ${ }^{\mathrm{b}}$, B. Bourbiaux ${ }^{\mathrm{a}}$ \\ ${ }^{a}$ IFP Energies nouvelles, Geosciences Division, France \\ ${ }^{b}$ IFP Energies nouvelles, Mechatronics and Numerics Division, France
}

\begin{abstract}
This paper proposes a robust methodology to calibrate steady-state models of foam flow through porous reservoirs from foam displacements on core samples. The underlying approach is an equivalence between foam mobility and foam lamellas density (or texture) at local-equilibrium. This calibration methodology is applied to foam displacements at different qualities and velocities on a series of sandstones. Its advantage lies in a deterministic transcription of flow measurements into texture data, by comparison with commonly-applied least-square-fit methods that may yield non unique solutions.

Scaling trends of foam parameters with porous medium permeability are then identified and discussed with the help of theoretical representations of foam flow in a confined medium. Although they remain to be further confirmed from other well-documented experimental data sets, these scaling laws can increase the reliability of reservoir simulators for the assessment of foam-based improved recovery processes in heterogeneous reservoirs.
\end{abstract}

Keywords: Enhanced Oil Recovery, Foam Modelling, Multiphase Flow, Capillary Pressure, Porous Media Permeability, Reservoir Simulation

\section{Introduction}

Since the sixties, foam-drive processes have been considered as a promising enhanced oil recovery (EOR) technique $[1,2,3,4,5]$. Indeed, the injection of foam instead of gas alleviates gravity override and detrimental effects of heterogeneities and viscous instabilities on displacement efficiency.

Foam in porous media is defined as a dispersion of gas in liquid carrying surfactants, such that at least a fraction of the gas phase is discontinuous and the liquid phase is continuous and connected through wetting films and lamellas separating gas bubbles. Surfactants in foam context are used to stabilize the thin liquid lamellas and promote the foaming ability of the mixture. Foam is usually characterized by its texture, defined as the number of foam bubbles or lamellas per unit volume of gas, and also its quality $f_{g}$ which is the ratio between the volumetric flux of foamed-gas and the total volumetric flux of gas and liquid.

\footnotetext{
${ }^{*}$ Corresponding author

Email address: frederic.douarche@ifpen.fr (F. Douarche)
}

Foam reduces dramatically gas mobility compared to gas flowing as a continuous phase, whereas the mobility of liquid phase is presumed to remain unchanged $[8,9,10]$. This reduced gas mobility can be seen as an increased effective gas viscosity, a decreased gas relative permeability, or also as a combination of the two effects $[11,12,13,14]$. Rheological properties of foam are complicated since they depend on several parameters such as foam texture, which is the result of several pore-level scale mechanisms of lamellas generation and destruction, gas and liquid velocities (foam behaves as a shear-thinning fluid in porous media), permeability and porosity of the porous medium, surfactant formulation and concentration. Coarsely-textured foams are characterized by a small number of lamellas and are referred to as weak foams since they provide a moderate gas mobility reduction, whereas finely-textured foams, called strong foams, are characterized by a large number of lamellas and reduce gas mobility remarkably. The transition from weak foam to strong foam state is called foam generation which is usually thought to be governed by pressure gradient (or equivalently total velocity) $[15,16,17,18]$.

The strong foam resulting from that generation pro-

June 2, 2017 


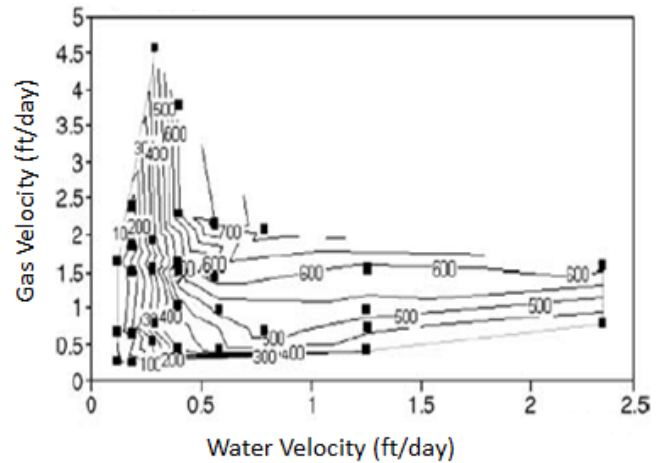

(a)

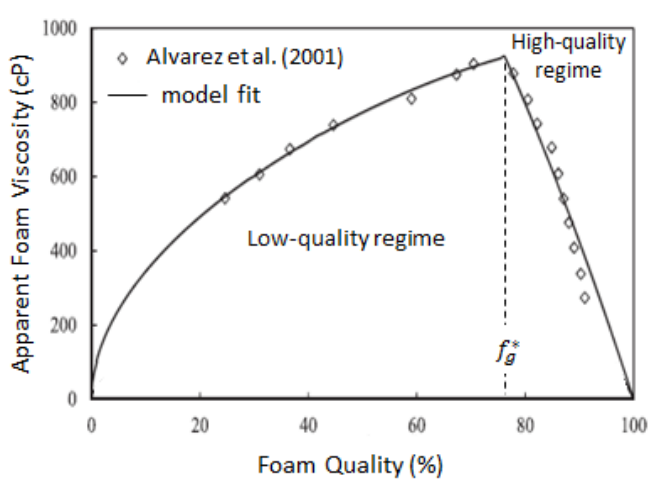

(b)

Figure 1: (a) Contours of iso-steady-state pressure gradient as a function of gas and liquid velocities: vertical and horizontal contours represent the high- and low-quality regimes, respectively (adapted from [6]); (b) Apparent foam viscosity for a single scan of foam quality at constant total velocity (adapted from [7]).

cess exhibits two different regimes: the low-quality regime, at which the steady-state pressure gradient is almost independent of water flow rate, and the highquality regime at which the pressure gradient is nearly independent of gas velocity. These two regimes were first highlighted by Osterloh and Jante [19] and later on by Alvarez et al [6] who represented the iso-value contours of the steady-state pressure gradient of strong foam as a function of gas and liquid volumetric fluxes as shown in Fig. 1(a). As any straight line drawn from the origin in this diagram represents a fixed foam quality, one can distinguish a specific value, called the optimal foam quality $f_{g}^{*}$, that divides the domain into two parts and provides the maximum pressure gradient that can be obtained for any given value of the total velocity. One can also clearly identify these two regimes from the evolution of the apparent foam viscosity $\mu_{f}$ with foam quality at constant total velocity, as reported in Fig. 1(b). $\mu_{f}$ is directly inferred from the pressure gradient $\nabla P$ by application of Darcy's law to foam considered as a single homogenous phase, that is $\mu_{f}=k|\nabla P| / u$, where $k$ is the permeability of the porous medium and $u$ the total velocity. In the low-quality regime, $\mu_{f}$ (or $\nabla P$ ) increases with foam quality $f_{g}$ until the optimal foam quality $f_{g}^{*}$ for which the maximum value of the apparent foam viscosity is reached. For foam qualities higher than $f_{g}^{*}$, lamellas rupture occurs and $\mu_{f}$ (or $\nabla P$ ) decreases, as reported in Fig. 1(b).

The two strong-foam regimes are dominated by different mechanisms: the low-quality regime is characterized by the mobilization of foam bubbles in proportion to foam quality (bubble size is nearly fixed), and the high-quality regime is characterized by lamellas coalescence as the liquid films in this regime become un- stable. By conducting foam flow experiments in beadpacks, Khatib et al [20] found that the transition between these two regimes corresponds to a maximum or critical gas-liquid capillary pressure, denoted $P_{c}^{*}$, above which foam collapses. Since capillary pressure is related to water saturation, there is a limiting water saturation $S_{w}^{*}$ corresponding to $P_{c}^{*}$. It has been shown that the magnitude of $P_{c}^{*}$, or equivalently $S_{w}^{*}$, varies with surfactant concentration, electrolyte concentration, gas flow rate and permeability [20]. The dependence of $P_{c}^{*}$ to the permeability is not yet clearly elucidated. Nonetheless, predicting the evolution of foam apparent viscosity with the permeability of the porous medium is of primary importance in evaluating foam process at the scale of a reservoir with permeability heterogenities.

The objective of this work is two-fold. Firstly, a novel methodology based on lamellas populationbalance (PB) model [21] is applied to calibrate an implicit-texture (IT) foam model from the apparent viscosity data measured on a natural porous medium with different foam qualities and flow velocities. Then, the parameters of the IT model calibrated for three cores of different permeabilities are analyzed in order to elucidate their scaling laws with respect to the permeability of the porous medium.

\section{Foam modelling in porous media}

We consider a model for a two-phase flow in a porous medium in the presence of foam. We distinguish two phases: an aqueous phase $w$ and a gas phase $g$. This flow is modified by the presence of foam. Modelling foam requires the presence of a surfactant, which is transported by the water phase, and which is described by 
an additional mass balance equation. The surfactant is either mobile or adsorbed on the rock.

It has been shown that the transport of liquid is not affected by the presence of foam $[22,23,10]$. On the opposite, the gas velocity is significantly reduced by the presence of foam. Thus, to describe the water and gas phases, we consider a black-oil model [24, 25] where the gas phase involves a modified velocity which will be denoted $\mathbf{u}_{g}^{f}$. The mass conservation equations read:

$$
\left\{\begin{array}{l}
\partial_{t}\left(\Phi \rho_{w} S_{w}\right)+\nabla \cdot\left(\rho_{w} \mathbf{u}_{w}\right)=q_{w} \\
\partial_{t}\left(\Phi \rho_{w} S_{w} C_{w}^{s}+(1-\Phi) \rho_{r} C_{r}^{s}\right)+\nabla \cdot\left(\rho_{w} \mathbf{u}_{w} C_{w}^{s}\right)=q_{w} C_{w}^{s} \\
\partial_{t}\left(\Phi \rho_{g} S_{g}\right)+\nabla \cdot\left(\rho_{g} \mathbf{u}_{g}^{f}\right)=q_{g}
\end{array}\right.
$$

where $\Phi$ is the rock porosity. For each phase denoted $i \in\{w, g\}, S_{i}$ is the saturation, $\rho_{i}$ the mass density and $q_{i}$ the source/sink term per unit volume of porous medium. $C_{w}^{s}$ stands for the flowing surfactant mass fraction in the water phase and $C_{r}^{s}$ for the adsorbed surfactant mass fraction on the rock with $\rho_{r}$ the rock mass density. Mobile and adsorbed surfactant mass fractions are related with an adsorption law such as the Langmuir isotherm [26, 27].

Under laminar flow conditions, the pure phase velocities in permeable porous media are governed by the generalized Darcy law:

$$
\mathbf{u}_{i}=-\frac{k k_{r i}}{\mu_{i}}\left(\nabla P_{i}-\rho_{i} \mathbf{g}\right)
$$

where $k$ is the rock permeability, $\mu_{i}$ the pure phase viscosity, $P_{i}$ the pressure of the phase $i$ and $\mathbf{g}$ the gravity. $k_{r i}$ is the relative permeability for the pure phase $i$, i.e. without lamellas. We suppose the relative permeabilities and capillary pressure functions are known, with given saturation end points. In our specific context of foam modelling, we will only consider the gas and water phases, thus $S_{g} \in\left[S_{g c}, 1-S_{w r}\right]$ where $S_{g c}$ is the connate gas saturation and $S_{w r}$ the residual water saturation.

In order to simplify the notations in the following, we introduce the phase mobility $\lambda_{i}=k_{r i} / \mu_{i}$ and the interstitial velocity $\mathbf{v}_{i}=\mathbf{u}_{i} /\left(\Phi S_{i}\right)$ (see [28] for more details). We also introduce the phase fractional flow $f_{i}=\left|\mathbf{u}_{i}\right| /|\mathbf{u}|$ where the total velocity $\mathbf{u}=\sum_{i \in\{w, g\}} \mathbf{u}_{i}$ is the sum of the phases velocities.

As mentioned earlier, the gas velocity is significantly reduced when foam develops. Hence, in the presence of foam, the liquid velocity reads $\mathbf{u}_{w}=$ $-k \lambda_{w}\left(\nabla P_{w}-\rho_{w} \mathbf{g}\right)$, while the Darcy's law for the gas phase is modified to account for the gas mobility reduc- tion and reads:

$$
\mathbf{u}_{g}^{f}=-k \lambda_{g}^{f}\left(\nabla P_{g}-\rho_{g} \mathbf{g}\right) \quad \text { with } \quad \lambda_{g}^{f}=\frac{k_{r g}^{f}}{\mu_{g}^{f}}
$$

where $\lambda_{g}^{f}$ is the gas mobility when foam is present, $k_{r g}^{f}$ and $\mu_{g}^{f}$ the relative permeability and the viscosity of the foamed-gas, respectively.

Let us consider now the case of a one-dimensional, horizontal and incompressible two-phase flow of gas and water. System given by Eqs 1 and 2 becomes:

$$
\partial_{t}\left(S_{g}\right)+\frac{u}{\Phi} \partial_{x}\left(f_{g}\right)=0 \quad \text { with } \quad f_{g}=\varphi_{g}+\psi_{g} \partial_{x}\left(S_{g}\right)
$$

where

$$
\varphi_{g}=\frac{\lambda_{g}}{\lambda_{g}+\lambda_{w}} \quad \text { and } \quad \psi_{g}=\frac{k}{u} \frac{\lambda_{g} \lambda_{w}}{\lambda_{g}+\lambda_{w}} \frac{d P_{c}}{d S_{g}}
$$

and $P_{c}=P_{g}-P_{w}$ denotes the gas-water capillary pressure. Whenever the capillary pressure derivative is small and/or the total velocity $u$ is high, the capillary pressure term $\psi_{g}$ is negligible and the fractional flow of gas reads:

$$
f_{g}=\frac{\lambda_{g}}{\lambda_{g}+\lambda_{w}}=\frac{1}{1+\frac{\mu_{g}}{\mu_{w}} \frac{k_{r w}}{k_{r g}}}
$$

When foam develops, Eqs 3 and 5 read:

$$
\begin{aligned}
& \partial_{t}\left(S_{g}\right)+\frac{u}{\Phi} \partial_{x}\left(f_{g}^{f}\right)=0 \\
& \text { with } f_{g}^{f}=\frac{\lambda_{g}^{f}}{\lambda_{g}^{f}+\lambda_{w}}=\frac{1}{1+\frac{\mu_{g}^{f}}{\mu_{w}} \frac{k_{r w}}{k_{r g}^{f}}}
\end{aligned}
$$

Eq. 6 is completed by a foam model that relates gas permeability and/or viscosity to some foam and flow parameters that are relevant for the model formulation. In the next section, we focus on a implicit-texture foam model.

In order to simplify the notations in the following, the gas or foamed-gas fractional flow function will be denoted $f_{g}$.

\section{Implicit-texture foam model}

Since a gas viscosity increase is equivalent to a decrease in the gas relative permeability term, most implicit-texture (IT) models include all foam effects, for the sake of simplicity, in the gas relative permeability term, such that the gas viscosity remains unchanged whatever foam is present or not, i.e. $\mu_{g}^{f}=\mu_{g}$. However, 
the gas relative permeability $k_{r g}$ is multiplied by a multiparameter interpolation function $F M$ including the contributions of physical parameters impacting the gas mobility reduction, such that $k_{r g}^{f}=F M k_{r g}$. The IT model of IFPEN PumaFlow simulator [29] is considered in this study for the only purpose of demonstrating the methodology of IT model calibration. Following PumaFlow IT model, $F M$ is given by

$$
F M=\frac{1}{1+\left(M_{\mathrm{ref}}-1\right) \prod_{i=1}^{4} F_{i}}
$$

where $M_{\text {ref }}$ is the reference gas mobility reduction under optimal conditions of the rock-fluid-additive system under consideration, and $F_{i}$ are functions of the parameters impacting foam performance that are surfactant concentration, water saturation, oil saturation and gas velocity, or equivalently the gas capillary number. In this paper, we focus on the effect of water saturation and gas velocity on foam performance. Thus, we only consider the so-called dry-out function $F_{2}$ and shear-thinning function $F_{4}\left(F_{1}\right.$ and $F_{3}$ functions accounting for the surfactant concentration and oil saturation effect are not developed herein). $F_{2}$ is given by

$$
F_{2}\left(S_{w}\right)=\frac{1}{2}+\frac{1}{\pi} \arctan \left[\Theta\left(S_{w}-S_{w}^{*}\right)\right]
$$

where $\Theta$ is the parameter governing the sharpness of transition from the high-quality to the low-quality regimes of strong-foam when the water saturation approaches the value $S_{w}^{*}$. A very high value of $\Theta$ (several thousands) leads to a steep transition and in this case foam coalescence occurs at the single water saturation $S_{w}^{*}$, whereas a low value (less than 100 for instance) leads to a smooth transition in the vicinity of $S_{w}^{*}$. As previously described, $S_{w}^{*}$ is close to the water saturation at which $P_{c}^{*}$ is reached and foam becomes subject to significant coarsening and collapsing. Note that $F_{2}$ function can be regarded also as an implicit description of the foam texture effects on gas mobility at local-equilibrium $[21,7]$. The shear-thinning function $F_{4}$ is defined as

$$
F_{4}\left(N_{c g}\right)=\left(\frac{N_{c g}^{\mathrm{ref}}}{N_{c g}}\right)^{e_{c}} \quad \text { with } \quad N_{c g}=\frac{\mu_{g} v_{g}^{f}}{\sigma}
$$

where $N_{c g}^{\text {ref }}$ is the reference capillary number for which $M_{\text {ref }}$ is reached, $v_{g}^{f}=u_{g}^{f} /\left(\Phi S_{g}\right)$ the interstitial foamedgas velocities, $\sigma$ the surface tension between gas and water in presence of surfactant and $e_{c}$ the shear-thinning exponent. The $F_{4}$ function definition remains controversial since some authors $[30,31,32]$ consider the total velocity instead of the gas velociy to define the capillary number.

\section{Foam displacements experimental data}

The experiments under consideration [33] consist in foam displacements in three Fontainebleau sandstone cores. The gas phase is composed of $80 \%$ of carbon dioxide and $20 \%$ of methane, and the aqueous phase of synthetic desulfated sea water (DSW), wherein a $5 \mathrm{~g} / \mathrm{L}$ surfactant is dissolved to generate foam. The thermodynamic conditions are $60{ }^{\circ} \mathrm{C}$ and 80 bar. For each of the three cores, a set of displacements was performed with different values of the foam quality. Each experiment at a given quality begins with the co-injection of brine and gas at different total flow rates respecting the selected quality value. The pressure drop is recorded at steady state at each selected total flow rate. The same injection sequence at a given quality and different total flow rates is then performed again, but with the surfactant solution instead of brine in order to generate foam. After the highest flow rate has been tested, foam is reinjected at the initial rate to measure the hysteresis of foam mobility. However, these hysteresis effects are not investigated herein. Table 1 summarizes the properties of the three cores used during this experimental study.

Table 1: Core samples properties [33].

\begin{tabular}{lllll}
\hline Core & $\begin{array}{l}\text { Diameter } \\
(\mathrm{cm})\end{array}$ & $\begin{array}{l}\text { Length } \\
(\mathrm{cm})\end{array}$ & $\begin{array}{l}\Phi \\
(\%)\end{array}$ & $\begin{array}{l}k \\
(\mathrm{mD})\end{array}$ \\
\hline 1 & 2.4 & 8.8 & 12 & 368 \\
2 & 2.4 & 13.1 & 11.7 & 121 \\
3 & 2.4 & 15.4 & 10.9 & 55 \\
\hline
\end{tabular}

As raw steady-state pressure drop measurements show fluctuations at low velocities, they were averaged to smooth unavoidable experimental fluctuations. Then, we infer the measured apparent foam viscosity $\mu_{f}^{\exp }$ as $\mu_{f}^{\exp }=\frac{k}{u} \frac{|\Delta P|}{L}$, where $\Delta P$ is the pressure drop measured at steady-state and $L$ the core length. Thus, for each core, $\mu_{f}^{\exp }$ is determined as a function of foam quality $f_{g}$ and total velocity $u$ and constitutes the main experiment outputs.

\section{IT foam model calibration}

Procedures to fit apparent foam viscosity $\mu_{f}^{\exp }$ as a function of foam quality have been provided by several authors [31, 34, 32, 35, 7]. Two methods will be applied and compared herein. The first one is based on the procedure proposed by Farajzadeh et al [34] that we have modified to account for the total velocity effects. The 
second proposed method is based on the equivalence between implicit-texture (IT) and population-balance (PB) foam models, such that the adjustment of the dry-out function $F_{2}$ and the shear-thinning function $F_{4}$ can be made separately. $F_{2}$ calibration is ensured directly from the experimental measurements transcribed into dimensionless foam texture and $F_{4}$ is estimated from flow ratescan experiments at low-quality regime. Each method will be applied for the three cores in order to investigate the effect of rock permeability on the foam model parameters. Conventional gas-water relative permeabilities are required and determined hereafter.

\subsection{Preliminary step: determination of conventional gas-water relative permeabilities}

This step is mandatory if conventional relative permeabilities are not available. Gas-water relative permeabilities were determined from the gas-water coinjection data measured with different flow rate ratios (or qualities), as described in Section 4. Only the steadystate measurements at the highest velocity are considered for the following reasons: (1) the magnitude of the measurements oscillation is reduced when increasing the velocity, and (2) the assumption of negligible capillary pressure is more relevant at high flow rate (see expression of $\psi_{g}$ in Eq. 4). Power laws were used to adjust the relative permeability functions, namely:

$$
k_{r g}=k_{r g}^{0}(1-S)^{n_{g}} \quad \text { and } \quad k_{r w}=k_{r w}^{0} S^{n_{w}}
$$

where $S=\left(S_{w}-S_{w r}\right) /\left(1-S_{w r}-S_{g c}\right)$ is the normalized water saturation, $S_{w r}$ the residual water saturation and $S_{g c}$ the connate gas saturation; $k_{r g}^{0}$ and $k_{r w}^{0}$ denote the gas and water maximum relative permeabilities, and $n_{g}$ and $n_{w}$ gas and water relative permeabilities exponents. Hence, the relative permeability determination problem involves six unknowns that are the water and gas saturation endpoints, maximum relative permeabilities and exponents, leading to the 6-tuple $X=\left(n_{g}, n_{w}, S_{w r}, S_{g c}, k_{r g}^{0}, k_{r w}^{0}\right)$.

These unknowns are determined by solving an optimization problem that consists in minimizing the sum of the squared differences between calculated and measured pressure drops at steady-state for each fractional flow value investigated during gas-water co-injection experiment. We note $\mathscr{F}_{\alpha}$ the set of fractional flow applied to the core sample $\alpha=1,2,3$. That is, for any core $\alpha$, we define the following objective function

$$
\begin{aligned}
& \mathscr{O}_{\alpha}^{k}(X)=\frac{1}{2} \sum_{f_{g} \in \mathscr{F}_{\alpha}}\left|\delta\left(f_{g}, X\right)\right|^{2} \\
& \text { with } \delta\left(f_{g}, X\right)=\frac{\Delta P^{\exp }\left(f_{g}\right)-\Delta P^{\mathrm{cal}}\left(f_{g}, X\right)}{\Delta P^{\exp }\left(f_{g}\right)}
\end{aligned}
$$

where, for any fractional flow $f_{g} \in \mathscr{F}_{\alpha}, \delta\left(f_{g}, X\right)$ is the relative error to measurement. $\Delta P^{\exp }\left(f_{g}\right)$ and $\Delta P^{\mathrm{cal}}\left(f_{g}, X\right)$ are the measured and the calculated pressure drops, respectively.

The minimum value of the objective function $\mathscr{O}_{\alpha}^{k}(X)$ is resolved on a discrete set $\mathscr{X}$ of the $k_{r}$ parameters. This space is finely sampled over sufficiently-large intervals: the lower and the upper bounds for each parameter were selected such that the results will be consistent with relative permeability fonctions of water-wet sandstones [36, 37, 38].

For any such parameters combination $X \in \mathscr{X}$, the pressure drop values are calculated in two steps. First, the gas saturation $S_{g}$, corresponding to the investigated gas fractional flow $f_{g} \in \mathscr{F}_{\alpha}$, is determined by inverting the gas fractional flow equation reformulated below with the relative permeability power functions:

$$
\begin{aligned}
& f_{g}\left(S_{g}\right)=\frac{1}{1+c \frac{\left(1-S_{g}-S_{w r}\right)^{n_{w}}}{\left(S_{g}-S_{g c}\right)^{n_{g}}}} \\
& \text { with } \quad c=\frac{\mu_{g}}{\mu_{w}} \frac{k_{r g}^{0}}{k_{r w}^{0}}\left(1-S_{g c}-S_{w r}\right)^{n_{g}-n_{w}}
\end{aligned}
$$

The steady-state pressure drop is then calculated by application of generalized Darcy law to any phase, for instance gas phase as follows:

$$
\Delta P^{\mathrm{cal}}\left(f_{g}, X\right)=\frac{\mu_{g} L f_{g} u}{k k_{r g}\left(S_{g}\right)}
$$

A unique set of $k_{r}$ parameters was determined for the first two cores of highest permeabilities and another set for the third one, because the porous structure differs between the first two cores and the third one according to capillary pressure data. Indeed, the mercury injection curves measured on companion plugs show that pore size distribution for Fontainebleau sandstone is modified for permeabilities in the order of a few tenths millidarcies or less, probably as a result of increased cementing. These mercury injection data were used to determine the gas-water capillary functions of our three samples as shown in Fig. 8(a). The third core capillary pressure curve differs significantly from the other two that are homothetic. Hence, a unique set of $k_{r}$ parameters was determined for the first two cores of highest permeabilities from the minimization of the sum $\mathscr{O}_{1}^{k}(X)+\mathscr{O}_{2}^{k}(X)$, and another set for the third one by minimizing $\mathscr{O}_{3}^{k}(X)$.

\subsection{Foam model calibration}

To start with, one determines the liquid saturation established for each foam displacement. Taking into account that the water relative permeability $k_{r w}$ remains 
unchanged in the presence of foam, $S_{w}$ is obtained by inverting the $k_{r w}$ function. Indeed, combining the equations of the apparent foam viscosity and the generalized Darcy's law applied to the water phase, one has $k_{r w}=\mu_{w}\left(1-f_{g}\right) / \mu_{f}^{\exp }\left(f_{g}, u\right)$. Replacing $k_{r w}$ by the power law function given by Eq. 7 yields the following expression for the liquid saturation $S_{w}$ :

$$
S_{w}=S_{w r}+\left(1-S_{w r}-S_{g c}\right)\left[\frac{\mu_{w}\left(1-f_{g}\right)}{k_{r w}^{0} \mu_{f}^{\exp }\left(f_{g}, u\right)}\right]^{1 / n_{w}}
$$

As evoked above, two methods will be applied herein in order to calibrate the IT foam model from the measurements. First, we present the direct calibration method proposed by Farajzadeh et al [34] which is based on a least-square minimization. Then, we propose our new mehodology that circumvents some difficulties in the calibration method.

\subsubsection{Farajzadeh et al method: adjustment by a least- square minimization}

The values of liquid saturation obtained in the second step are used in the definition of an objective function $\mathscr{O}^{F M}$ that minimizes the differences between the foam apparent viscosity values determined from experimental data and the ones calculated according to the IT foam model. Using generalized Darcy equations and the modified $k_{r g}$ function of IT model, the calculated apparent foam viscosity for each point can be derived from the following equation:

$$
\mu_{f}^{\mathrm{cal}}\left(Y ; f_{g} u\right)=\frac{1}{\frac{k_{r w}\left(S_{w}\right)}{\mu_{w}}+\frac{F M\left(Y ; S_{w}, f_{g} u\right) k_{r g}\left(S_{w}\right)}{\mu_{g}}}
$$

where $S_{w}$ is computed from the experimented $f_{g}$ with Eq. 8 and

$$
F M\left(Y ; S_{w}, f_{g} u\right)=\frac{1}{1+\left(M_{\mathrm{ref}}-1\right) F_{2}\left(S_{w}\right) F_{4}\left(f_{g} u\right)}
$$

where $Y=\left(M_{\mathrm{ref}}, S_{w}^{*}, \Theta, e_{c}\right)$ is the 4-tuple composed of the IT foam model parameters to be adjusted. The constraints on parameters are needed to be set at physical limits, namely $M_{\text {ref }} \geq 0, S_{w r} \leq S_{w}^{*} \leq 1-S_{g c}, \Theta>0$ and $e_{c} \geq 0$. In addition, the reference capillary number $N_{c g}^{\text {ref }}$ is set at the minimum reached value of the gas capillary number during the experimental study reached in steady-state, such that

$$
N_{c g}^{\mathrm{ref}}=\min _{\alpha \in\{1,2,3\}}\left(\min _{f_{g} \in \mathscr{F}_{\alpha}, u \in \mathscr{U}_{\alpha}} \frac{\mu_{g} f_{g} u}{\sigma \Phi S_{g}}\right)
$$

The objective function $\mathscr{O}^{F M}(Y)$ to be minimized was defined for each core as:

$$
\begin{gathered}
\mathscr{O}_{\alpha}^{F M}(Y)=\frac{1}{2} \sum_{f_{g} \in \mathscr{F}_{\alpha}} \sum_{u \in \mathscr{U}_{\alpha}}\left|\delta\left(Y ; f_{g} u\right)\right|^{2} \\
\text { with } \delta\left(Y ; f_{g} u\right)=\frac{\mu_{f}^{\exp }\left(f_{g}, u\right)-\mu_{f}^{\mathrm{cal}}\left(Y ; f_{g} u\right)}{\mu_{f}^{\exp }\left(f_{g}, u\right)}
\end{gathered}
$$

where for any core $\alpha$, for any experimental foam quality $f_{g}$ and any experimental total velocity $u, \delta\left(Y ; f_{g} u\right)$ is the relative error on the measured apparent foam viscosity. The objective function is minimized using a constrained least-square algorithm. The constraints are in form of lower and upper bounds on each foam model parameter of the set $Y$.

\subsubsection{Identification method between $I T$ and $P B$ foam models}

The identification method is based on the equivalence between IT and PB foam models at local-equilibrium, as previously demonstrated by the authors [21]. The mechanistic PB models take into account of the dependence of gas mobility on foam texture through an effective gas viscosity, that is expressed as: $[11,39,13,12]$

$$
\mu_{g}^{f}=\mu_{g}+\frac{C_{f} n_{f}}{\left(v_{g}^{f}\right)^{c}}
$$

where the constant $C_{f}$ varies with the porous medium permeability and the surfactant [40], $n_{f}$ is the lineic foam texture, $v_{g}^{f}$ the foamed-gas interstitial velocity, and $c$ an exponent that expresses the shear-thinning behavior of foam at fixed foam texture. That viscosity law results from an extension of the equation of gas bubble motion in a tube proposed by Bretherton [41] to a train of bubbles or lamellas [11]. These theoretical studies indicate that the value of $c$ is close to $\frac{1}{3}$ at lower velocities. The exact value of the shear-thinning exponent $c$ remains controversial in the literature due to the complexity of foam flow in natural porous media that can hardly be assimilated to smooth parallel capillary tubes.

However, this rheological law allows to relate the saturation (characteristic parameter of multiphase flow in porous media) to foam texture (characteristic parameter of foam) in steady-state. The relationship between these two parameters is obtained by including the expression of $\mu_{g}^{f}$ given by Eq. 10 in the fractional flow equation of foamed-gas. After rearranging the terms, we obtain:

$$
C_{f} n_{f}=\left(\frac{1-f_{g}}{f_{g}} \frac{k_{r g}\left(S_{w}\right)}{k_{r w}\left(S_{w}\right)} \mu_{w}-\mu_{g}\right)\left(\frac{f_{g} u}{\Phi S_{g}}\right)^{c}
$$

Taking into account water saturation given by Eq. 8, Eq. 11 shows that a single value of foam texture $n_{f}$ can 
be determined for any value of foam quality $f_{g}$ and of total velocity $u$.

In view of the independence of the parameter $C_{f}$ on foam flow parameters, one can calculate the dimensionless foam texture values $n_{D}$ for each set of experiments as:

$$
n_{D}\left(f_{g}, u\right)=\frac{C_{f} n_{f}\left(f_{g}, u\right)}{\max _{f_{g}^{\prime} \in \mathscr{F}_{\alpha}, u^{\prime} \in \mathscr{U}_{\alpha}} C_{f} n_{f}\left(f_{g}^{\prime}, u^{\prime}\right)}
$$

Then, the parameter $C_{f}$ can be estimated from the maximum value of the product $C_{f} n_{f}$ which is, in this case, equal to $C_{f} n_{f}^{\max }$, where $n_{f}^{\max }$ is the reference foam texture value. $n_{f}^{\max }$ is correlated to the average pore radius $r$ of the porous medium under consideration which can be estimated by $r=\sqrt{8 k / \Phi}$, following the simplified representation of the porous medium as a bundle of identical capillary tubes with a radius $r . n_{f}^{\max }$ definition has to be specified further. Indeed, the pressure gradient due to foam flow is related to the number of lamellas per unit length in the flow direction under consideration, therefore the foam texture to be considered in Eq. 10 is a lineic texture, i.e. the number of lamellas per unit flow length. Then, considering foam bubbles as spheres of radius equal (or proportional) to pore radius $r$, the number of lamellas per unit flow length within pores of radius $r$ is equal to $\frac{\pi r^{2}}{\frac{4}{3} \pi(\lambda r)^{3}}=\frac{3}{4 \lambda^{3} r} \sim \frac{1}{r}$, where $\lambda$ is a proportionality constant between the bubble and the pore radius. For model calibration developed later on, $\lambda$ will be arbitrarily taken equal to 1 (spherical bubbles of radius $r$ ). Finally the maximum texture $n_{f}^{\max }$ also denoted $n_{L}^{\max }$, will be quantified as:

$$
n_{f}^{\max }=n_{L}^{\max }=\frac{3}{4} \sqrt{\frac{\Phi}{8 k}}
$$

The experimental flow conditions for which the maximum texture is reached are referred to as reference conditions.

Then, the foamed-gas mobility expression according to the IT model is identified to the mobility expression derived from the PB model in order to calibrate the constants and functions of the IT model as detailed in [21]. That identification leads to the following equalities:

$$
\left\{\begin{aligned}
e_{c} & =c \\
M_{\mathrm{ref}} & =1+\frac{C_{f} n_{f}^{\max }}{\mu_{g}}\left(\frac{\mu_{g}}{N_{c g}^{\mathrm{ref}} \sigma}\right)^{c} \\
F_{2} & =n_{D} \\
F_{4} & =\left(\frac{N_{c g}^{\mathrm{ref}}}{N_{c g}}\right)^{c}
\end{aligned}\right.
$$

where $N_{c g}^{\text {ref }}$ is the minimum of the values obtained by considering the Eq. 9. The parameter $M_{\text {ref }}$ can be easily determined from the physical parameters $C_{f}$ and $n_{f}^{\max }$, characteristic of the rock-fluid-additive system under consideration. However, the calibration of $F_{2}$ and $F_{4}$ functions is not straightforward and needs to be detailed further in the following.

Calibration of $F_{4}$ function. The shear-thinning exponent $c$ is determined from flow-rate-scan experiment at the low-quality regime. Combining the expression of the apparent foam viscosity $\mu_{f}=k|\nabla P| / u$, the generalized Darcy equations for water and gas, and Eq. 10, we can express $\mu_{f}$ as:

$$
\begin{aligned}
\mu_{f} & =\left[\mu_{g}+\frac{C_{f} n_{f}^{\max } n_{D}\left(S_{w}\right)}{\left(\frac{\sigma N_{c g}}{\mu_{g}}\right)^{c}}\right] \frac{f_{g}}{k_{r g}\left(S_{w}\right)} \\
& \approx \frac{C_{f} n_{f}^{\max } n_{D}\left(S_{w}\right)}{\left(\frac{\sigma N_{c g}}{\mu_{g}}\right)^{c}} \frac{f_{g}}{k_{r g}\left(S_{w}\right)}
\end{aligned}
$$

That apparent foam viscosity formula is an approximation that ignores liquid slugs contribution to the apparent viscosity and considers that the continuous-gas viscosity value is negligible compared to that of foamedgas. Considering that the texture function $n_{D}$ does not account for fluid velocities effects, $n_{D}\left(S_{w}\right)$ can be fixed for a given foam quality. Thus, we conclude that $\mu_{f} k_{r g}\left(S_{w}\right) \sim\left(N_{c g}\right)^{-c}$ for a flow-rate-scan experiment at a given quality within the low-quality regime (excluding coalescence regime at the highest qualities). Thus, for any such experiment, the values of the shear-thinning exponent $c$ can be determined from the fit of $\mu_{f} k_{r g}\left(S_{w}\right)$ data to a power function of $N_{c g}$. As the fitted $c$ value does not much vary from one quality to another, an average value can be adopted, as shown later on.

Calibration of $F_{2}$ function. The dry-out function is identified to the dimensionless foam texture $n_{D}$, that is, the parameters $\Theta$ and $S_{w}^{*}$ can be determined by adjusting $n_{D}$. Alternatively, one can determine graphically these two parameters plotting $n_{D}\left(S_{w}\right)$. First, $S_{w}^{*}$ is identified as the inverse of $n_{D}(S w)$ function for a dimensionless texture equal to $\frac{1}{2}$. Once $S_{w}^{*}$ is identified, the parameter $\Theta$ can be determined by setting $F_{2}=1$ at the reference water saturation $S_{w}^{\text {ref }}$ (saturation data corresponding to the maximum foam texture). As $F_{2}$ is defined as an arctan function, a tolerance $\varepsilon$ on $F_{2}\left(S_{w}^{\text {ref }}\right)$ is considered such that $F_{2}\left(S_{w}^{\text {ref }}\right)=1-\varepsilon$, hence $\Theta=\tan \left[\pi\left(\frac{1}{2}-\varepsilon\right)\right] /\left(S_{w}^{\text {ref }}-S_{w}^{*}\right)$. The latter graphical method is accurate if the dimensionless foam texture 
$n_{D}$ data covers the transition range between low- and high-quality regimes. Otherwise, $S_{w}^{*}$ and $\Theta$ can only be roughly estimated and a least-square method may be used to further adjust those parameters.

\section{Results and discussions}

\subsection{Adjustment of conventional gas-water fractional flow}

By following the method detailed in Section 5.1, we calibrate the end-points and exponents of the gaswater relative permeability functions. To that end, the two optimal parameter sets that minimize the objective functions $\mathscr{O}_{1}^{k}+\mathscr{O}_{2}^{k}$ and $\mathscr{O}_{3}^{k}$ as defined in Section 5.1 , are searched on a discrete space defined by $n_{g} \in$ $[1.3,2.8]$ with $\Delta n_{g}=0.1, n_{w} \in[2.8,4.2]$ with $\Delta n_{w}=$ $0.1, S_{w r} \in[0,0.4]$ with $\Delta S_{w r}=0.05, S_{g c} \in[0,0.2]$ with $\Delta S_{g c}=0.05, k_{r w}^{0} \in[0.1,0.5]$ with $\Delta k_{r w}^{0}=0.05$ and $k_{r g}^{0} \in$ $[0.6,0.9]$ with $\Delta k_{r g}^{0}=0.05$. The optimal sets are listed in Table 2, and the corresponding gas-water relative permeabilities and fractional flow curves are plotted in Fig. 2. The corresponding steady-state pressure drops are reported in Fig. 3.

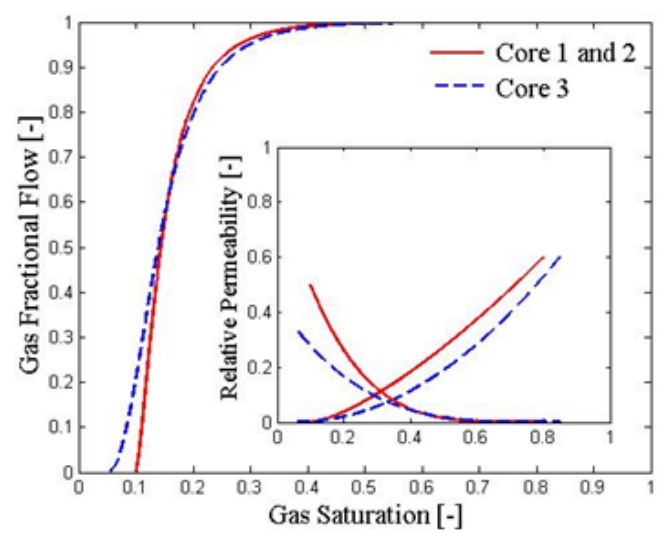

Figure 2: Adjusted gas fractional flow curves and relative permeability functions for cores 1 and 2, and core 3 (optimal parameters are reported in Table 2).

\subsection{Calibration of IT foam model parameters}

On one hand, we begin with Farajzadeh et al method (see Section 5.2.1). The set of foam model parameters, denoted $Y$, is adjusted using a least-square method applied over a discrete domain of unknown parameters limited by lower bound $\left(10^{2}, 10, S_{w r}, 0.1\right)$ and the upper bound $\left(10^{4}, 10^{4}, S_{w r}+0.3,0.8\right)$. The IT foam model parameters calibrated with that method are given in Table 4. On the other hand, we apply the method of identification of IT foam model to PB model. We start with the determination of the shear-thinning exponent. For this purpose, the products $\mu_{f} k_{r g}\left(S_{w}\right)$ at different total flow velocities and given quality are adjusted to a power law function with an exponent equal to the shearthinning parameter according to previous developments (see Section 5.2.2). As shown in Fig. 4, adjusted $c$ values are very close and an average $c$ value can be retained. Averaged $c$ values are also very close from one core to another, as reported in Table 4; as a consequence we did not attempt to identify any scaling law.

Then, we proceed with the calibration of the dry-out function $F_{2}$. To that end, the $C_{f} n_{f}$ values calculated using Eq. 11 are plotted against foam quality, in order to determine the maximum $C_{f} n_{f}$ product, that is equal to $C_{f} n_{f}^{\max }$ since $C_{f}$ is invariant for a given foam-rock system. The maximum foam texture $n_{f}^{\max }$ is estimated from the characteristic pore size of the core under consideration (estimated itself from its porosity and permeability as specified before). Results are shown in Fig. 5. The dimensionless texture $n_{D}$ can then be calculated and drawn versus foam quality, or versus saturation by taking into account the bi-univocal relationship between $f_{g}$ and $S_{w}$ resulting from Eq. 8. The so-determined $n_{D}\left(S_{w}\right)$ function can then be used to calibrate the constants $S_{w}^{*}$ and $\Theta$ of the dry-out function $F_{2}$. A least-square method of adjustment was used and applied to the sole experimental data at low velocity that best reflects the texture effects and are also less dispersed (see Fig. 6). Indeed, the IT foam model implicitly assumes that $F_{2}$, and in particular $S_{w}^{*}$, is independent of flow rate whose effect is accounted for by function $F_{4}$. Invariant $S_{w}^{*}$ is also consistent with the concept of fixed limiting capillary pressure $P_{c}^{*}$ as explained by Zhou and Rossen [42]. To end with the IT-PB identification method, the reference gas mobility reduction, $M_{\text {ref }}$ is calculated from Eq. 14 . To summarize, the above calibration sequence turns out to be an almost-fully deterministic treatment procedure of experimental data based on a transcription of apparent viscosity measurements in terms of both saturation and texture. Of course, the resulting foam model remains an approximate model, which is inherent to the usual noise observed on foam flow data and to the underlying assumptions of the foam flow model, such as the absence of any capillary effects.

The steady-state PB model parameters obtained from the identification method are mentioned in Table 3 and the calibrated IT foam model parameters using the two methods are reported in Table 4.

The predicitive capacity of the IT model calibrated before was tested from the comparison of both the foamed-gas fractional flow and the apparent foam vis- 
Table 2: Optimal $k_{r}$ functions for both cores 1 and 2, and core 3 .

\begin{tabular}{lllllll}
\hline core & $n_{g}(-)$ & $n_{w}(-)$ & $S_{w r}(-)$ & $S_{g c}(-)$ & $k_{r g}^{0}(-)$ & $k_{r w}^{0}(-)$ \\
\hline 1 and 2 & 1.4 & 4.2 & 0.2 & 0.1 & 0.6 & 0.5 \\
3 & 2.0 & 3.6 & 0.15 & 0.05 & 0.6 & 0.35
\end{tabular}

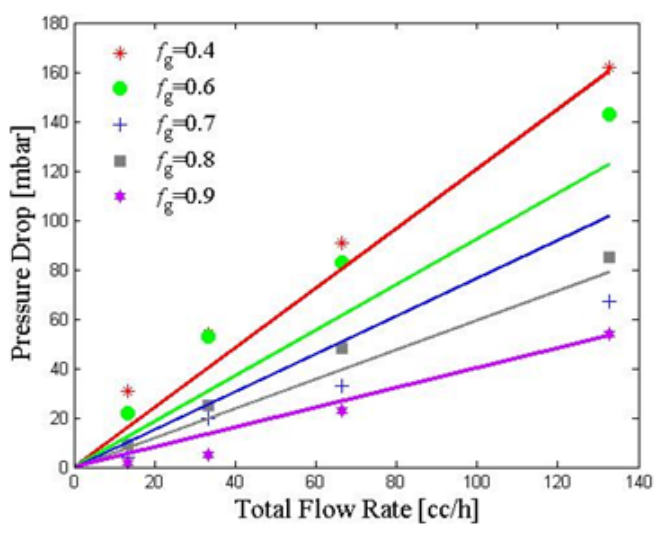

(a)

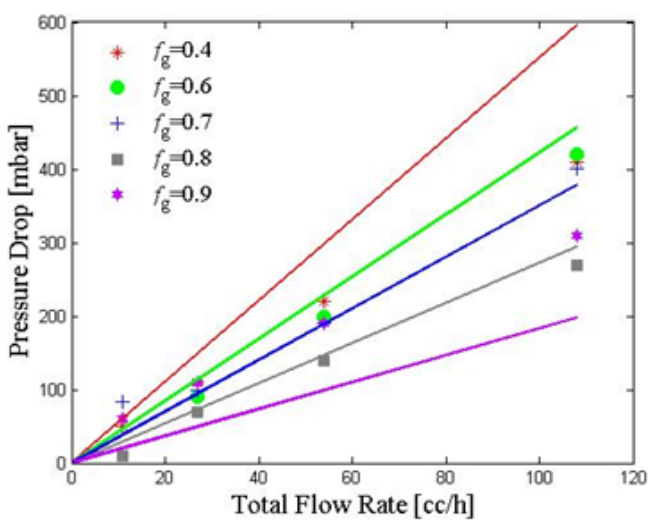

(b)

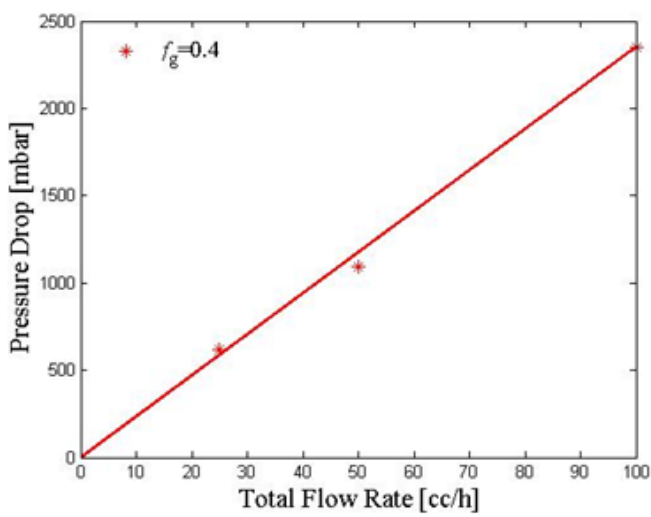

(c)

Figure 3: Comparison between the calculated/measured steady-state pressure drops after adjustment of the relative permeabilities with respect to the higher total flow rate in each case: (a) core 1, (b) core 2 and (c) core 3 (lines and symbols represent the calculation and the measurements, respectively).

Table 3: Adjusted steady-state PB model parameters with respect to apparent foam viscosity measurements. The constant $c$ shown in the unit of $C_{f}$ is the calibrated shear-thinning exponent for each core.

\begin{tabular}{lll}
\hline Core & $C_{f}\left(\mathrm{~m}^{1+c}\right.$. Pa.s $\left.^{1-c}\right)$ & $n_{f}^{\max }\left(\mathrm{m}^{-1}\right)$ \\
\hline 1 & $3.56 \times 10^{-10}$ & $1.52 \times 10^{5}$ \\
2 & $1.42 \times 10^{-10}$ & $2.63 \times 10^{5}$ \\
3 & $0.498 \times 10^{-10}$ & $3.75 \times 10^{5}$ \\
\hline
\end{tabular}

cosity calculated with that model, with the experimental data. First, we solve analytically the fractional flow equation obtained by expressing the foam mobility in
Eq. 6 with the IT-model formulation, that is

$$
f_{g}\left(Y ; S_{g}\right)=\frac{1}{1+\frac{\mu_{g}}{\mu_{w}} \frac{k_{r w}\left(S_{g}\right)}{F M\left(Y ; f_{g}, S_{g}\right) k_{r g}\left(S_{g}\right)}}
$$

where $Y$ is one of the two sets of IT-model parameters given in Table 4. Indeed, the foamed-gas fractional flow versus gas saturation was constructed by finely discretizing the variation interval $] 0,1\left[\right.$ of $f_{g}$ and calculating for each point the corresponding gas saturation $S_{g}$ by inversing Eq. 15 . Then, we use the relation $f_{g}$ $S_{g}$ in order to compute the IT-model apparent viscosity from $\frac{1}{\mu_{f}}=\frac{k_{r w}}{\mu_{w}}+\frac{F M k_{r g}}{\mu_{g}}$. Comparison results are shown 


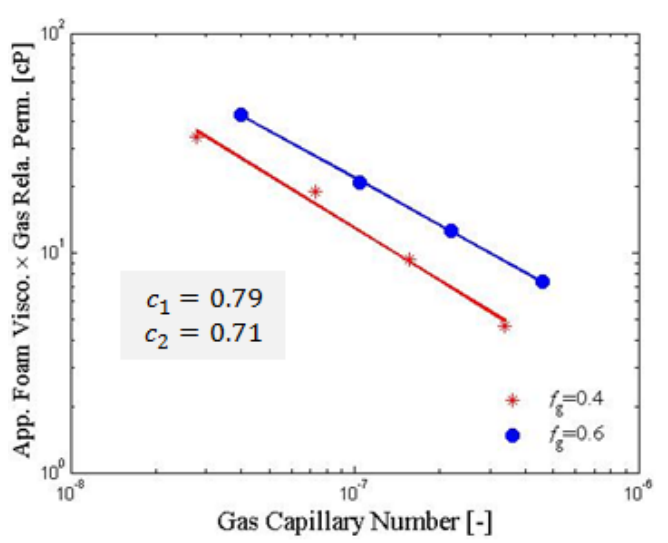

(a)

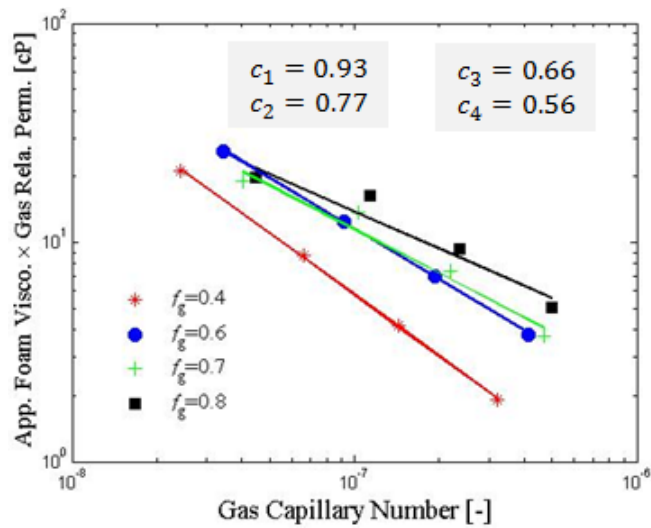

(b)

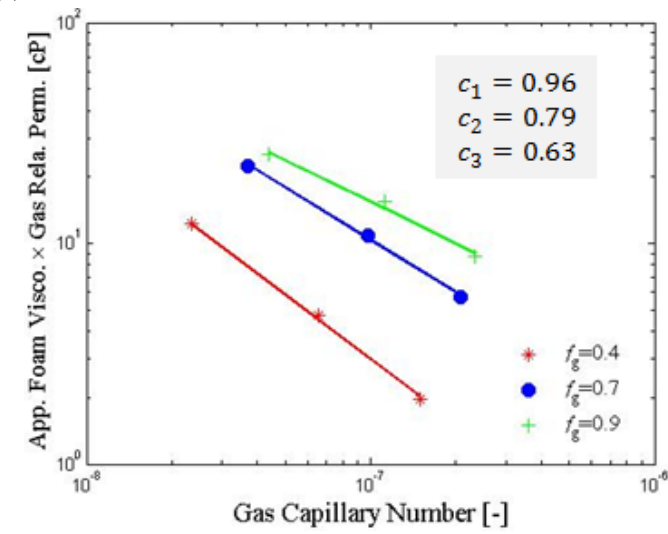

(c)

Figure 4: Adjustment of flow rate-scan experiments at low-quality regime by power laws in order to determine the shear-thinning exponent: (a) core 1 , (b) core 2 and (c) core 3 . The lines are the final adjusted power laws and the symbols the experimental data. $c_{i}$ values shown in sub-figures correspond to the adjusted exponents for each foam quality. The shear-thinning exponent $c$ for each core is calculated from the average of the $c_{i}$ values.

Table 4: Adjusted foam model parameters with respect to experimental data obtained from both Farajzadeh et al [34] method and identification method.

\begin{tabular}{llllll}
\hline Core & $M_{\text {ref }}(-)$ & $S_{w}^{*}(-)$ & $\Theta(-)$ & $N_{c g}^{\text {ref }}(-)$ & $c=e_{c}(-)$ \\
\hline \multicolumn{5}{l}{ Farajzadeh et al method } \\
\hline 1 & $4.342 \times 10^{3}$ & 0.3409 & 424 & $2.774 \times 10^{-8}$ & 0.72 \\
2 & $2.983 \times 10^{3}$ & 0.3918 & 308 & $2.774 \times 10^{-8}$ & 0.76 \\
3 & $2.055 \times 10^{3}$ & 0.2782 & 549 & $2.774 \times 10^{-8}$ & 0.67 \\
\hline \multicolumn{7}{l}{ IT-PB identification } & & & & \\
\hline 1 & $5.317 \times 10^{3}$ & 0.3232 & 36 & $2.774 \times 10^{-7}$ & 0.75 \\
2 & $3.002 \times 10^{3}$ & 0.3816 & 34 & $2.774 \times 10^{-7}$ & 0.73 \\
3 & $2.736 \times 10^{3}$ & 0.3016 & $10^{3}$ & $2.774 \times 10^{-7}$ & 0.79 \\
\hline
\end{tabular}

in Fig. 7. The comparison is shown for both models calibrated with either the least-square method or the PB-IT identification method. We found that the fractional flow, the maximum apparent foam viscosities and the transi- tion between low- and high-quality regimes are fairlywell matched for the two first cores, whereas for the third one, the low-quality regime is hardly predicted. This issue is clear on Fig. 6(c) since it shows that the 


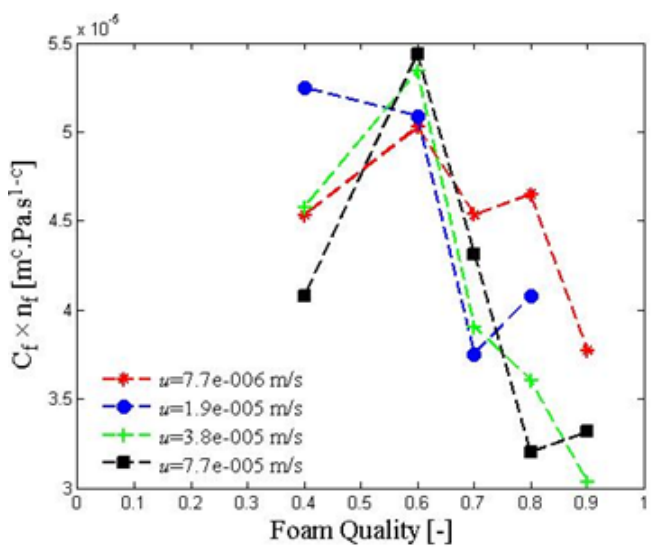

(a)

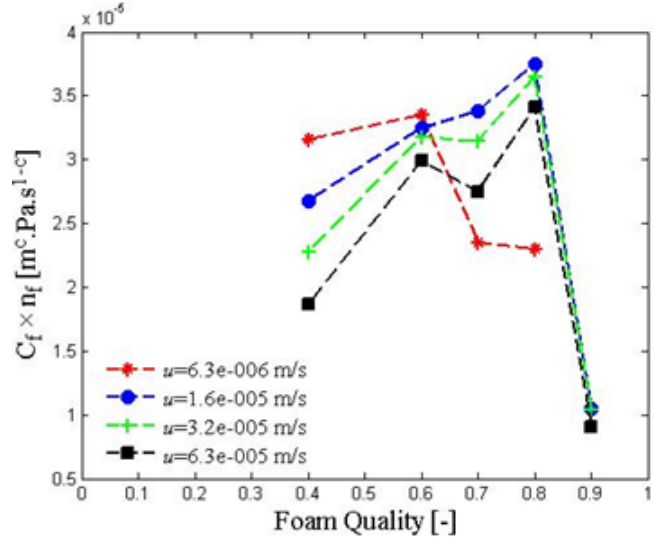

(b)

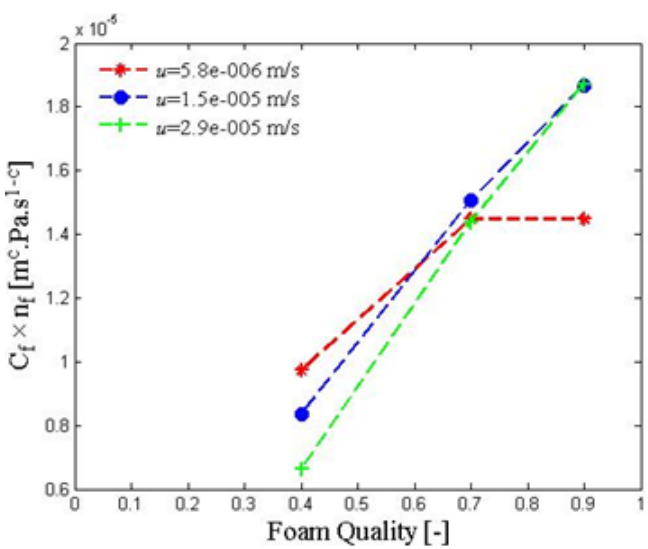

(c)

Figure 5: Variation of the product $C_{f} n_{f}$ versus foam quality: (a) core 1, (b) core 2 and (c) core 3 (dashed lines represent visual guides for the variation at constant total velocity and symbols the measurements).

foam texture decreases with water saturation in the lowquality regime, which is unexpected, and consequently cannot be captured by the $F_{2}$ function of an IT foam model that assumes a constant foam texture in the lowquality regime. Due to the poor consistency of core 3 data, some reserves will be taken in the scaling of foam model parameters with respect to the permeability, as discussed in the last section of that paper.

\subsection{Effect of permeability on foam model parameters}

Foam generation, propagation and destruction mechanisms that determine its texture and mobility are influenced by the pore size distribution, characterized by the capillary pressure of the porous medium through which foam is flowing. In this regard, IT foam model parameters, specifically $S_{w}^{*}$ (or equivalently $P_{c}^{*}$ ), $\Theta$ and $M_{\text {ref }}$, are believed to vary with the porous medium. This issue is addressed in the literature [20, 34, 43]; qualitative trends are only reported due to the difficulties in acquiring consistent and complete data sets to constrain models. In this context, we investigate the impact of the porous medium, essentially characterized by its permeability (or its capillary pressure), on IT model parameterization by interpreting the results of model calibration for the reviewed cores. Porosity does not vary by more than $1 \%$, hence it has been discarded in the analysis presented hereafter. Furthermore, our analysis is mainly based on the results of the PB-IT identification method. The impact of flow velocity conditions seems to be independent of the porous medium properties according to the obtained $c$ exponent values which are very closed from one core to another, as previously discussed in Section 6.2. Thus the scaling of the function $F_{4}$ parameters is not studied in the following. 


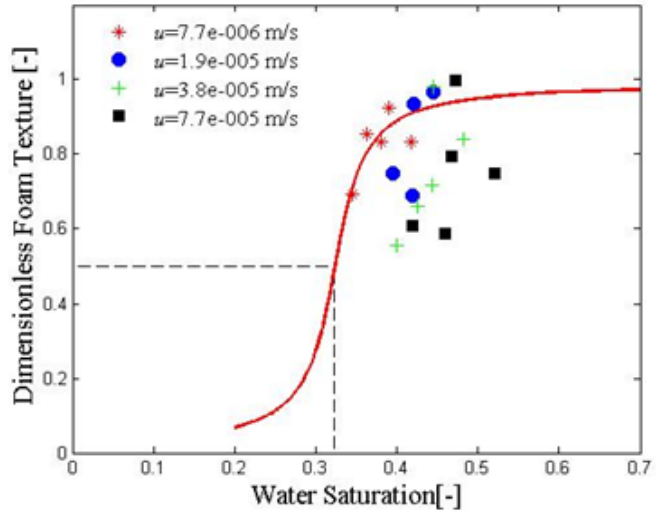

(a)

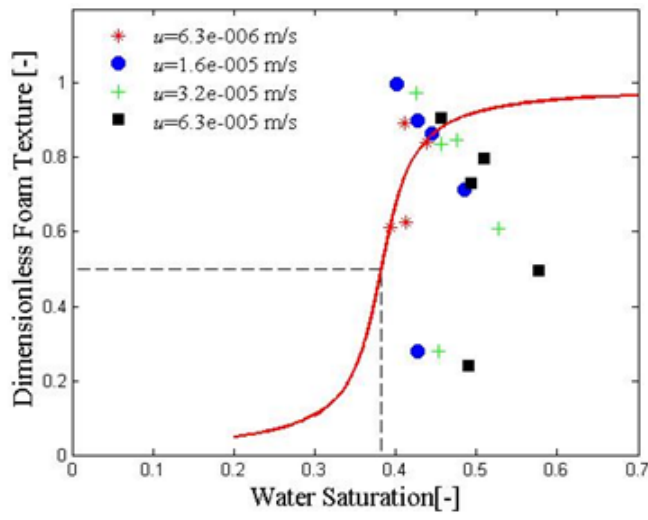

(b)

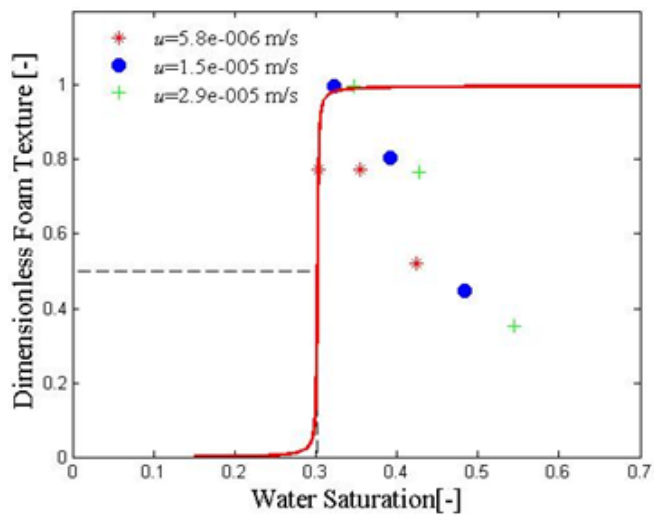

(c)

Figure 6: Variation of dimensionless foam texture versus water saturation as inferred from the identification method: (a) core 1, (b) core 2 and (c) core 3 . The continuous lines represent the adjusted $F_{2}$ function with respect to the lowest velocity and the points are the experimental data.

\subsubsection{Effect of permeability on critical saturation $S_{w}^{*}$ and critical capillary pressure $P_{c}^{*}$}

The dependence of critical saturation $S_{w}^{*}$ on the porous medium properties can be analyzed in terms of capillary pressure due to the univocal relationship between saturation and capillary pressure. To that end, one has to specify those $P_{c}\left(S_{w}\right)$ functions for the the three cores under consideration. Different flow behaviors between the first two cores and the third one lead us suspect that the latter had a different porous structure. This was acknowledged by the $P_{c}$ data measured on similar plugs respectively of $50 \mathrm{mD}$ and $465 \mathrm{mD}$, that are found not to obey the same dimensionless J-function. Then the $P_{c}$ functions of cores 1 and 2 were determined from the same J-function derived from the $465 \mathrm{mD}$-plug $P_{c}$ data and the the $P_{c}$ function of core 3 from the J-function derived from the $50 \mathrm{mD}$-plug $P_{c}$ data. These $P_{c}$ curves are given in Fig. 8(a).

Let us now examine the evolution of $S_{w}^{*}$ between the three cores. Table 4 shows an increase of $S_{w}^{*}$ when the permeability decreases for cores 1 and 2 that have the same porous structure (i.e. homothetic, following the same $\mathrm{J}$-function). That result is consistent with the observations from other authors, Farajzadeh et al [34], Khatib et al [20]) and Kapetas et al [43]. Interpretation of such result is proposed as follows.

A quasi-static viewpoint of foam flow at low velocities is adopted. This leads us to assume that foam coalescence starts when the saturation corresponds to a fixed critical value of the capillary pressure, that is equal to the characteristic disjoining pressure of the foam under consideration, whatever the porous medium properties. Retaining that for a fixed- $P_{c}^{*}$ assumption for given foam, the inversion of $P_{c}$ function for $P_{c}^{*}$ leads to a $S_{w}^{*}$ value that necessarily increases for homothetic porous media of decreasing permeability (like cores 1 and 2). The reason is that (a) gas-water $P_{c}$ is a monotonous decreasing function of $S_{w}$, and (b) $P_{c}$ increases when the permeability of homothetic cores decreases. We tried to verify if previous interpretation was consistent with the 

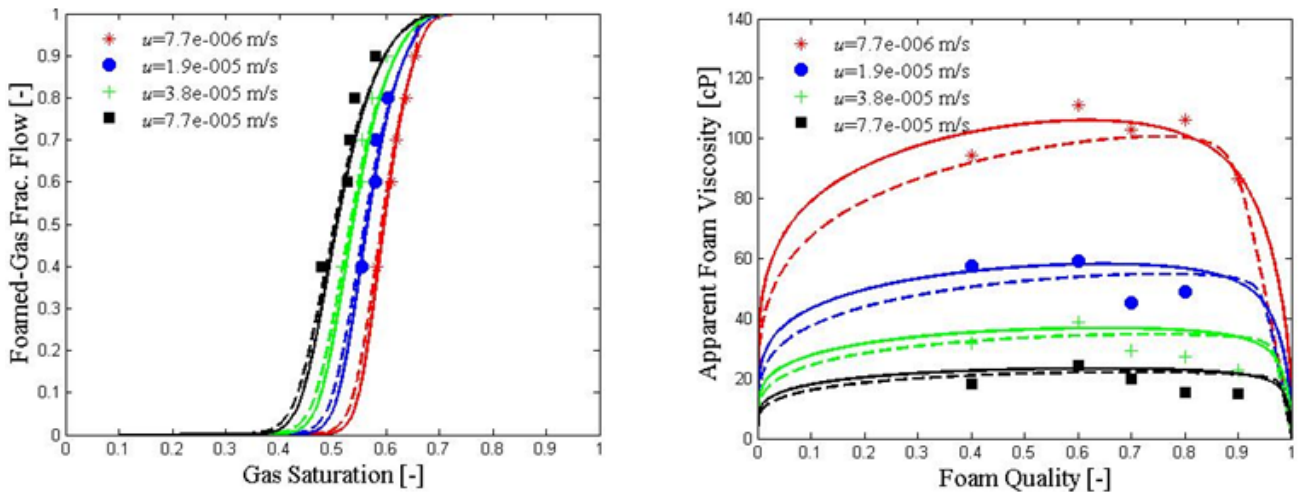

(a)
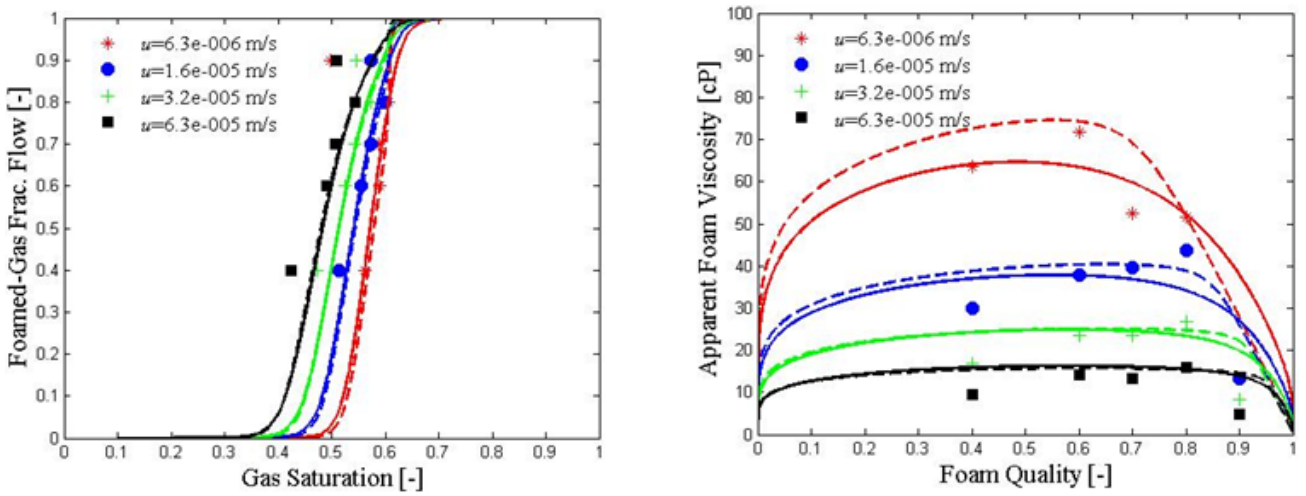

(b)
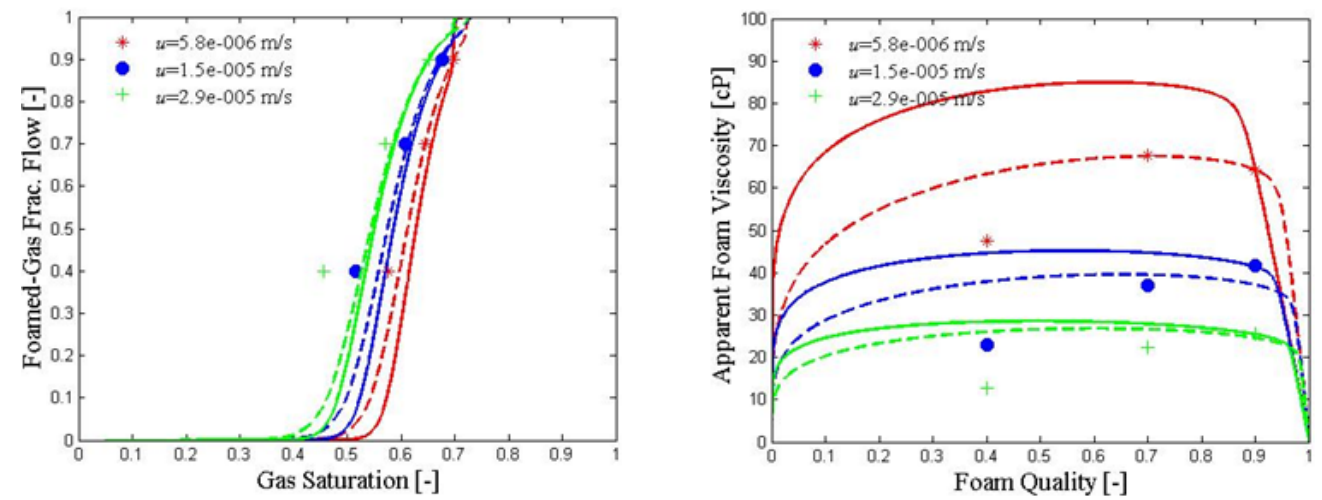

(c)

Figure 7: Comparison between both the computed foamed-gas fractional flow and the apparent foam viscosity, and the experimental data: (a) core 1, (b) core 2 and (c) core 3. The lines represent the computed results (dashed lines for Farajzadeh et al method and solid lines for the PB-IT identification method) and the symbols the experimental data.

$S_{w}^{*}$ values calibrated for homothetic cores 1 and 2 . To that end, we determined $P_{c}^{*}$ as the $P_{c}$ value of core 1 at the critical saturation (given in Table 4) then inverted the $P_{c}$ function of core 2 for that fixed $P_{c}^{*}$ value. Unfortunately, the $S_{w}^{*}$ determined this way for core 2 was about 0.83 which is much higher than the actually-calibrated value of Table 4 . Finally, it turns out that a fixed- $P_{c}^{*}$ assumption would explain the trend of evolution of $S_{w}^{*}$ with permeability, but is not valid from a quantitative standpoint.

Let us then examine the evolution of the critical capillary pressure with the permeability. $P_{c}^{*}$ values were 
simply determined from the $P_{c}$ curves of Fig. 8(a) for the calibrated $S_{w}^{*}$ values of Table 4 . The resulting $P_{c}^{*}$ values for the 3 cores are shown in Fig. 8(b) as a function of permeabillity. $P_{c}^{*}$ is found to decrease with increasing permeability. An evolution close to $1 / \sqrt{k}$ is found as in several published studies from Khatib et al [20] and Farajzadeh et al [34].

Interpretation of such an evolution can be attempted from a dynamic standpoint. We have to consider the conditions of rupture of films flowing in pores. Film rupture occurs dynamically through a shear process when the transverse gradient of local velocity within pore, denoted $d w / d y$ (derivative of the local velocity within a pore with respect to distance $y$ from pore axis), exceeds a given threshold value $w_{\max }^{\prime}$ that is a characteristic of the foam under consideration. For a laminar (Poiseuille) flow of gas in a capillary tube of radius $r$, $d w / d y$ is maximum at pore wall and equal to $\frac{r}{2 \mu}|\nabla P|$. Therefore, the limit of stability of films is reached for a pressure gradient $|\nabla P|_{\max }$ equal to $2 \mu w_{\max }^{\prime} / r$, i.e. inversely proportional to characteristic pore radius $r$. To that maximum pressure gradient corresponds a maximum value of the dynamic capillary pressure within the porous medium, called the critical capillary pressure $P_{c}^{*}$, that is also inversely proportional to $r$ or $\sqrt{k}$. Finally, the dynamic viewpoint of film stability explains the observed evolution of $P_{c}^{*}$ with the permeability. However, a scaling of $P_{c}^{*}$ as $1 / \sqrt{k}$ would imply that $S_{w}^{*}$ remains constant for homothetic porous media, whereas an increase of $S_{w}^{*}$ with decreasing permeability, although moderate, is acknowledged. Eventually, neither the quasi-static (fixed $P_{c}^{*}$ ) interpretation model nor the dynamic (fixed $S_{w}^{*}$ ) one explain both $S_{w}^{*}$ and $P_{c}^{*}$ evolutions with $k$. The reality seems to lie between these two models but looks closer to the dynamic one, such that $P_{c}^{*} \sim 1 / k^{\alpha}$ with $\alpha \leq \frac{1}{2}$.

In order to develop the equivalent scaling law of the limiting water saturation, we write the capillary pressure as [44] $P_{c}(S)=P_{e} S^{-1 / \lambda}$, where $P_{e}$ is the the entry capillary pressure, $S$ the normalized water saturation and $\lambda$ the pore size distribution index $(\lambda=3$ in our case). Then, the relation between $S_{w}^{*}$ and $P_{c}^{*}$ is obtained by inverting the $P_{c}$ function at $P_{c}^{*}$, leading to $S_{w}^{*}=S_{w r}+\left(1-S_{w r}-S_{g c}\right)\left(P_{c}^{*} / P_{e}\right)^{-\lambda}$. Inserting the scaling law of $P_{c}^{*}$ in the latter expression of $S_{w}^{*}$ and knowing that $P_{e} \sim k^{-0.5}$ yields $S_{w}^{*} \sim k^{\lambda(\alpha-0.5)}$. Thus, when $\alpha<0.5$, the limiting water saturation (as the limiting capillary pressure) increases with decreasing permeability. In our case, $S_{w}^{*} \sim k^{-0.3}$ for the two homothetic cores.

Table 5 summarizes all the films stability standpoints discussed above and the resulting parameters evolution with permeability. Undoubtedly, the problem is probably more complex and cannot be reduced to the simple analysis attempted above. As indicated by Rossen and Zhou [45], the texture of foams changes under critical conditions, and hysteresis and gas trapping (Chen [46]) renders the analysis still more complex. Definitely, further research work is required to clarify the flow characteristics under these so-called critical conditions, on the basis of well-documented experimental data that cover the whole transition in foam flow regime.

\subsubsection{Evolution of transition parameter $\Theta$ with perme- ability}

Results from Table 4 would indicate that the transition parameter $\Theta$ increases when $k$ decreases as found also by other authors [34]. However, if we only consider the two homothetic cores, the parameter $\Theta$ appears to be invariant. In any case, explaining such evolution is dubious and still being debated in the literature. We propose herein a simple graphical method to construct scaling law for the parameter $\Theta$ with permeability in the case of homothetic porous media. We assume a destabilization pressure zone of lamellas which can be centered on $P_{c}^{*}$ values and bounded by two parallel lines following the observed scaling law of $P_{c}^{*}$, as illustrated in Fig. 9. Thus, we can determine for each permeability the saturation interval $I$ over which the transition occurs by identifying the intersection between the $P_{c}$ curve and the destabilization zone. The transition interval $I$ can be expressed as $I=\left[1-S_{w}^{\text {opt }}, 1-S_{w}^{\mathrm{min}}\right]$, where $S_{w}^{\mathrm{min}}$ is the water saturation below which foam does no more exist, or equivalently $F_{2}\left(S_{w}^{\min }\right)=\varepsilon$ where $\varepsilon$ is the tolerance, and $S_{w}^{\text {opt }}$ the water saturation above which low-quality regime is reached (i.e. $F_{2}\left(S_{w}^{\text {opt }}\right)=1-\varepsilon$ ). Expressing $F_{2}$ function at $S_{w}^{\mathrm{min}}$ and $S_{w}^{\text {opt }}$ shows that the product $I \Theta$ is invariant and does not depend on the permeability. Thus, parameter $\Theta$ can be obtained from $\Theta \sim \frac{1}{I(k)}$. In particular, if the transition interval $I$ is narrower when the permeability decreases, $\Theta$ would increase with decreasing permeability. Application to the data of cores 1 and 2 yields $\Theta_{2}=\Theta_{1} \times I_{1} / I_{2}=36 \times 0.14 / 0.15=33.6$, which is very close to the adjusted value of $\Theta_{2}$ (cf. Table 4).

On the other hand, if we assume a constant destabilization pressure interval of lamellas $J$ centered on $P_{c}^{*}(k)$ such as $J=\left[P_{c}^{*}(k)-\Delta P_{c}, P_{c}^{*}(k)+\Delta P_{c}\right]$, where $\Delta P_{c}$ is the capillary pressure increment, we obtain the following relationship that governs the variation of $\Theta$ between two homothetic cores of permeability $k_{1}$ and $k_{2}$ (with $k_{2}<k_{1}$ ), porosity $\Phi_{1}$ and $\Phi_{2}$, and capillary pressure $P_{c, 1}$ and $P_{c, 2}$ : 


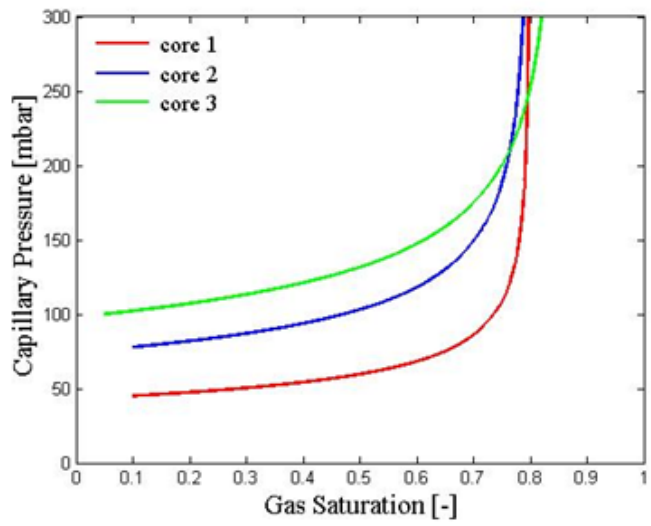

(a)

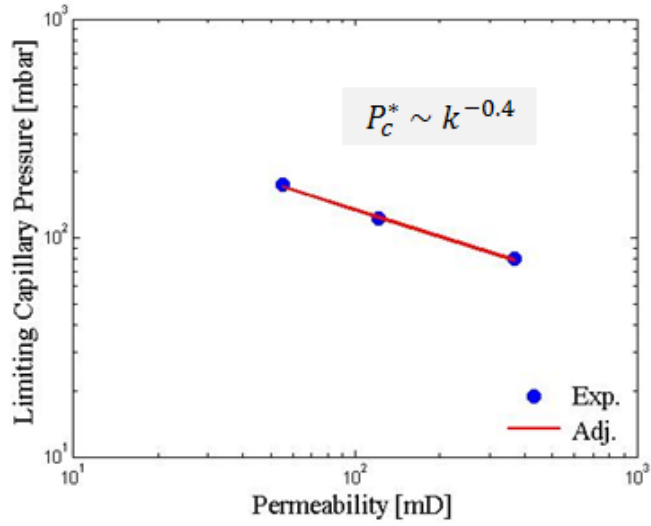

(b)

Figure 8: (a) Capillary pressure curves used for each core; (b) Effect of permeability on the limiting capillary pressure $P_{c}^{*}$. The points are estimated from the water saturation $S_{w}^{*}$ given in Table 4 using the identification method and the line is a power law adjustment. The optimal adjustment yields $P_{c}^{*} \sim k^{-0.4}$

Table 5: Scaling law trends recapitulation.

\begin{tabular}{||c|c|c||}
\hline $\begin{array}{c}\text { Theoretical model } \\
\text { for films stability }\end{array}$ & $\begin{array}{c}\text { Expected } P_{c}^{*} \\
\text { evolution with } k\end{array}$ & $\begin{array}{c}\text { Expected } S_{w}^{*} \\
\text { evolution with } k\end{array}$ \\
\hline \hline Static picture & Constant $P_{c}^{*}$ & $S_{w}^{*}$ increase with $k$ decrease \\
\hline \hline Dynamic picture & $P_{c}^{*} \sim 1 / \sqrt{k}$ & Constant $S_{w}^{*}$ \\
\hline \hline Observation & $\begin{array}{c}P_{c}^{*} \sim 1 / k^{\alpha} \text { with } \\
\alpha \leq \frac{1}{2}\end{array}$ & $\begin{array}{c}\text { Moderate } S_{w}^{*} \text { increase } \\
\text { with } k \text { decrease as } S_{w}^{*} \sim k^{\lambda(\alpha-0.5)}\end{array}$ \\
\hline
\end{tabular}

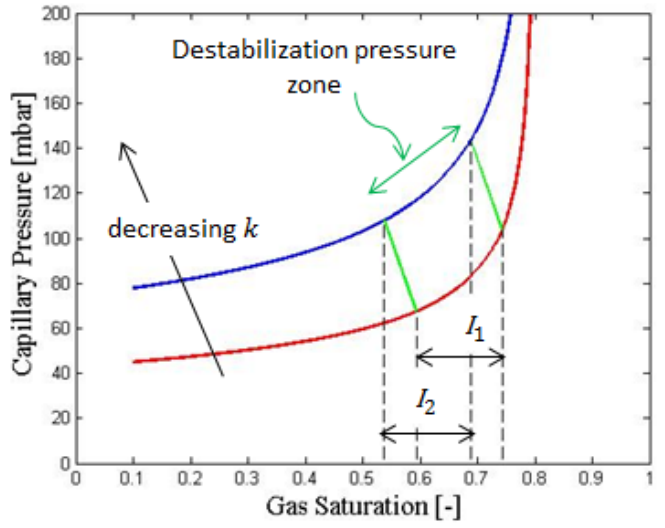

Figure 9: Graphical method to determine the parameter $\Theta$ as a function of the permeability for homothetic cores. The green lines represent the destabilization pressure zone of lamellas. $I_{1}$ and $I_{2}$ are the transition interval of saturation for the first permeability $k_{1}$ and the second permeability $k_{2}$ (with $k_{2}<k_{1}$ ), respectively. The transition parameter $\Theta$ for each core can be estimated as $\Theta_{1} \sim \frac{1}{I_{1}}$ and $\Theta_{2} \sim \frac{1}{I_{2}}$ (see the text).

$$
\Theta_{2} \frac{\mathrm{d} P_{c, 1}}{\mathrm{~d} S_{w}}\left(S_{w, 1}^{*}\right)=\Theta_{1} \frac{\mathrm{d} P_{c, 2}}{\mathrm{~d} S_{w}}\left(S_{w, 2}^{*}\right)
$$

where $S_{w, 1}^{*}$ and $S_{w, 2}^{*}$ are the limiting water saturation of core 1 and core 2, respectively. Application of Eq. 16 to predict $\Theta_{2}$ in our case gives $\Theta_{2}=37$ which is still acceptable value comparing to the adjusted one. Note that in the specific case of $P_{c}^{*} \sim \frac{1}{\sqrt{k}}$ and small porosity variations, the transition parameter $\Theta$ scales roughly as $\Theta \sim \frac{1}{\sqrt{k}}$.

\subsubsection{Effect of permeability on $M_{\text {ref }}$}

For a given foam, the mobility reduction $M_{\text {ref }}$ represents the ratio between the continuous gas mobility $k_{r g} / \mu_{g}$ and the foamed-gas mobility that is defined as $k_{r g}^{f} / \mu_{g}$ in IT models, or as $k_{r g} / \mu_{g}^{f}$ in PB models, at optimal foaming conditions. Scaling $M_{\text {ref }}$ for different porous media characterized by the same $k_{r g}$ functions can then be reduced to the scaling of $\mu_{g}^{f}$. Analogy between flow in a capillary tube (originally formulated by Bretherton [41]) and flow in a porous medium has led most authors to use the empirical rheological law given by Eq. 10 for foamed-gas. Eq. 14 for $M_{\text {ref }}$ tells us that, for a given velocity, the mobility reduction is at the 
first order determined by the product of constant $C_{f}$ and maximum texture $n_{f}^{\max }$.

Kovscek and Bertin [40] derived an expression of $\mu_{g}^{f}$ scaled with respect to rock permeability and capillary pressure by establishing an equivalence between rheological law given by Eq. 10 and Hirasaki and Lawson's formula [11] for the apparent viscosity of a train of bubbles of pre-determined volume flowing in a capillary tube, that is:

$$
\mu_{g}^{f}=0.85 \mu_{w} \frac{n_{L}}{r_{c}}\left(r^{2}+r_{c}^{2}\right)\left(\frac{\sigma}{3 \mu_{w} v_{g}^{f}}\right)^{\frac{1}{3}}
$$

where $r$ is the tube radius and $r_{c}$ the curvature radius of the Plateau borders separating gas bubbles. That formula also assumes that the surface tension gradient effects on effective gas viscosity are negligible, as well as the impact of water slugs when touching bubbles are flowing through the capillary. Actually, that expression of the effective viscosity can be considered for scaling purpose, because it is related to the geometrical characteristics of capillary tubes, or to the properties of the equivalent porous medium represented as a bundle of such capillary tubes. So that, we consider again the above formula; however, our analysis differs from the development made by Kovscek and Bertin.

For a given foam flowing in a porous medium of permeability $k$ and porosity $\Phi$, capillary tube radius $r$ is equivalent to the quantity $\sqrt{8 k / \Phi}$ and $r_{c}$ is related to the disjoining pressure of the films of the foam under consideration [47]. $\quad r_{c}$ is therefore assumed invariant for the same foam displaced through different porous media. Furthermore, we assume that $r_{c}$ is negligible compared to pore radii (such an assumption may not be valid however in very-low-permeability media). Therefore, $\mu_{g}^{f}$ scales as $n_{L} r^{2}$ under given velocity conditions. For porous media of different permeabilities with similar porosities, $r$ scales as $\sqrt{k}$ and the lineic foam texture, $n_{L}$, quantified as $\frac{3}{4 r}$ (see Section 5.2.2), scales as $1 / \sqrt{k}$, hence $\mu_{g}^{f}$ scales as $\sqrt{k}$. To end with, above analysis indicates that $M_{\text {ref }}$ is expected to increase as the square root of permeability for porous media of different permeabilities. That scaling approximation neglects small porosity variations compared to permeability variations in many natural porous media.

Analysis of $M_{\text {ref }}$ results for homothetic samples 1 and 2 is consistent with the previous relationship. Indeed, for homothetic cores 1 and 2, Table 4 indicates that, according to our modelling methodology by ITPB models identification, $M_{\mathrm{ref}(2)}=3002$ is very close to $M_{\text {ref(1) }} \sqrt{k_{2} / k_{1}}=5317 \times \sqrt{121 / 368}=3049$. The low- permeability core 3 is characterized by a porous structure slightly different from that of cores 1 and 2 . Its $M_{\text {ref }}$ value is also lower than the values for cores 1 and 2 but scaling permeability exponent differs from $\frac{1}{2}$. The dependence of the parameter $M_{\text {ref }}$ to the permeability $k$, as obtained from Table 4, is shown in Fig. 10.

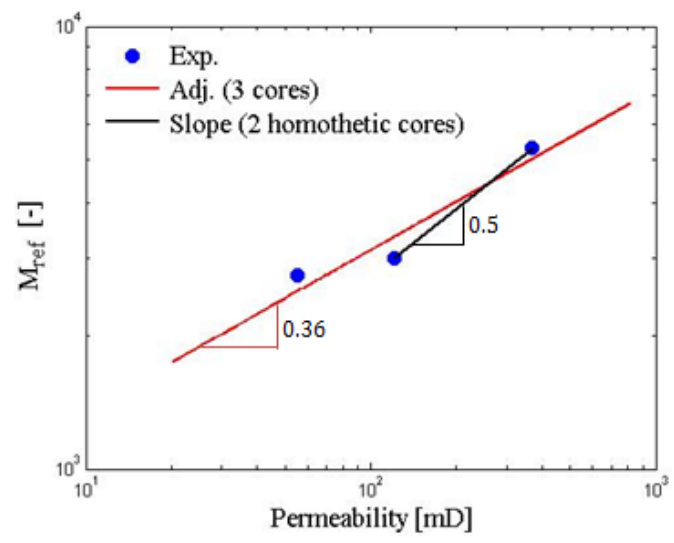

Figure 10: Dependence of $M_{\text {ref }}$ to the permeability $k$. Note that the adjustment includes three cores although the third core has a slightly different porous structure. The optimal adjustment yields $M_{\text {ref }} \sim k^{0.36}$. The data of the homothetic cores 1 and 2 show that $M_{\text {ref }} \sim \sqrt{k}$.

To conclude, the evolution of foam model parameters with porous medium properties seems to follow the scaling laws derived from an equivalence between foam flow in a porous medium and foam flow in capillary tubes. Such results are worth being investigated further on the basis of more extensive data set acquired on wellcharacterized porous media. Indeed, scaling foam performance with respect to rock properties is an important reservoir engineering issue because foam processes are primarily dedicated to reservoirs where the detrimental impact of heterogeneities on displacement has to be reduced.

\section{Conclusions}

A new methodology based on an equivalence with a foam texture model has been implemented to calibrate the steady-state implicit-texture model to predict foam flow effects on gas mobility in reservoir simulators. This deterministic calibration approach was applied to foam flow data measured on a series of sandstones of different permeabilities, both for demonstration of the advantage in terms of solution unicity, and for elucidating the scaling of foam flow model parameters with respect to porous medium properties. The following main conclusions can be drawn: 
(a) An IT foam model can be calibrated in a deterministic way to fit experimental steady-state apparent viscosity data, including the transition between the two low-quality and high-quality regimes. Calibration may however remain approximate for incomplete or scattered data sets such as the one acquired on the low-permeability core under consideration in this study.

(b) Scaling trends of foam parameters with porous medium permeability were found consistent with other published observations and interpreted:

- The shear-thinning function that models the flow velocity impact on foam mobility appears to be fairly invariant for cores of different permeabilities, with a characteristic exponent equal to about 0.7 .

- For homothetic porous media, the critical water saturation $S_{w}^{*}$ increases slightly when the permeability decreases; accordingly, the capillary pressure increases according to a law close to $1 / \sqrt{k}$ but maybe not exactly if one considers the $S_{w}^{*}$ change. Interpretation of such results were attempted thanks to two static and dynamic viewpoints of foam bubbles coalescence.

- The transition between low-quality and highquality regimes seems to be more abrupt in low-permeability cores than in highpermeability cores. Different explanations have been provided herein on such observation.

- The main result is a scaling of the mobility reduction ratio $M_{\text {ref }}$ as the square root of permeability. This scaling law was inferred from an analogy between foam flow in porous media and foam flow in capillary tubes, and was found consistent with the modelling of available experimental data on three cores, and with other experimental findings.

To conclude, we dispose of a robust procedure to analyze and model foam flow measurements in porous media. The scaling trends derived from the analysis of available data require to be further confirmed and extended to natural porous media of different structures and properties, with the help of other extensivelydocumented and accurate experimental data sets. However, they represent a first encouraging step for the assessment and selection of a foam-based process for improved oil recovery purposes.

\section{Acknowledgements}

The authors thank L. Nabzar and L. G. Pedroni for useful discussions and IFPEN for permission to publish this work.

\section{References}

[1] D. G. Bond, O. C. Holbrook, Gas drive oil recovery, Patent no. US Pat 2866507 (1958).

[2] L. W. Holm, Foam injection test in the Siggins field, Illinois, Society of Petroleum Engineers 22 (1970).

[3] T. M. Jonas, S. I. Chou, S. L. Vasicek, Evaluation of a $\mathrm{CO}_{2}$ foam field trial: Rangely Weber Sand Unit, Society of Petroleum Engineers (1990).

[4] J. P. Heller, D. A. Boone, R. J. Watts, Field test of $\mathrm{CO}_{2}$ mobility control at Rock Creek, Society of Petroleum Engineers, Paper SPE-14395-MS (1995)

[5] J. J. Sheng, Foams and their applications in enhancing oil recovery, in: Enhanced Oil Recovery Field Case Studies, Elsevier, 2013, pp. 251-280.

[6] J. M. Alvarez, H. J. Rivas, W. R. Rossen, Unified model for steady-state foam behavior at high and low foam qualities, SPE Journal 6 (2001) 325-333.

[7] M. Lotfollahi, R. Farajzadeh, M. Delshad, A. Varavei, W. R. Rossen, Comparison of implicit-texture and population-balance foam models, Journal of Natural Gas Science and Engineering 31 (2016) 184-197.

[8] G. Bernard, W. L. Jacobs, Effect of foam on trapped gas saturation and on permeability of porous media to water, Society of Petroleum Engineers Journal 5 (1965) 295-300.

[9] J. B. Lawson, J. Reisberg, Alternate slugs of gas and dilute surfactant for mobility control during chemical flooding, in: SPE/DOE Enhanced Oil Recovery Symposium, 1980.

[10] F. Friedmann, W. H. Chen, P. A. Gauglitz, Experimental and simulation study of high-temperature foam displacement in porous media, SPE Reservoir Engineering 6 (1991) 37-45.

[11] G. J. Hirasaki, J. B. Lawson, Mechanisms of foam flow in porous media: Apparent viscosity in smooth capillaries, Society of Petroleum Engineers Journal 25 (1985) 176-190.

[12] A. H. Falls, G. J. Hirasaki, T. W. Patzek, D. A. Gauglitz, D. D. Miller, T. Ratulowski, Development of a mechanistic foam simulator: The population balance and generation by snap-off, SPE Reservoir Engineering 3 (1988) 884-892.

[13] A. R. Kovscek, C. J. Radke, Fundamentals of foam transport in porous media, in Foams: fundamentals and applications in the petroleum industry, American Chemical Society Advances in Chemistry (M. J. Comstock and L. L. Schramm Eds) (1994).

[14] A. R. Kovscek, T. W. Patzek, C. J. Radke, A mechanistic population balance model for transient and steady-state foam flow in Boise sandstone, Chemical Engineering Science 50 (1995) 3783-3799.

[15] W. R. Rossen, P. A. Gauglitz, Percolation theory of creation and mobilization of foams in porous media, AIChE Journal 36 (1990) 1176-1188.

[16] Z. F. Dholkawala, H. K. Sarma, S. I. Kam, Application of fractional flow theory to foams in porous media, Journal of Petroleum Science and Engineering 57 (2007) 152-165.

[17] P. A. Gauglitz, F. Friedmann, S. I. Kam, W. R. Rossen, Foam generation in homogeneous porous media, Chemical Engineering Science 57 (2002) 4037-4052.

[18] M. Lotfollahi, I. Kim, M. R. Beygi, A. J. Worthen, C. Huh, K. P. Johnston, M. F. Wheeler, D. A. DiCarlo, Foam generation hys- 
teresis in porous media: Experiments and new insights, Transport in Porous Media 116 (2017) 687-703.

[19] W. T. Osterloh, M. J. Jante, Effects of gas and liquid velocity on steady-state foam flow at high temperature, Society of Petroleum Engineers (1992).

[20] Z. I. Khatib, G. J. Hirasaki, A. H. Falls, Effects of capillary pressure on coalescence and phase mobilities in foams flowing through porous media, SPE Reservoir Engineering 3 (1988) 919-926.

[21] O. Gassara, F. Douarche, B. Braconnier, B. Bourbiaux, Equivalence between implicit-texture and population-balance foam models, submitted to Transport in Porous Media (2017).

[22] G. Bernard, W. L. Jacobs, Effect of foam on trapped gas saturation and on permeability of porous media to water, SPE Journal 5 (1965) 295-300.

[23] J. B. Lawson, J. Reisberg, Alternate slugs of gas and dilute surfactant for mobility control during chemical flooding, SPE/DOE Enhanced Oil Recovery Symposium, Tulsa, Oklahoma (1980).

[24] D. W. Peaceman, Fundamentals of numerical reservoir simulation, volume 6 of Developments in Petroleum Science, Elsevier Science, Amsterdam, 1977.

[25] J. A. Trangenstein, J. B. Bell, Mathematical structure of the black-oil model for petroleum reservoir simulation, SIAM J. Appl. Math. 49 (1989) 749-783.

[26] L. W. Lake, Enhanced oil recovery, Prentice Hall, 1989.

[27] D. W. Green, G. P. Willhite, Enhanced oil recovery, volume 6 of SPE Textbook Series, Henry L. Doherty Memorial Fund of AIME, Society of Petroleum Engineers, 1998.

[28] C. M. Marle, Multiphase flow in porous media, third ed., Gulf Publishing Company, 1981.

[29] B. Braconnier, E. Flauraud, Q. L. Nguyen, Efficient scheme for chemical flooding simulation, Oil \& Gas Science and Technology - Revue d'IFP Energies nouvelles 69 (2014) 585-601.

[30] Y. Zeng, A. Muthuswamy, K. Ma, Le Wang, R. Farajzadeh, M. Puerto, S. Vincent-Bonnieu, A. A. Eftekhari, Y. Wang, C. Da, J. C. Joyce, S. L. Biswal, G. J. Hirasaki, Insights on foam transport from a texture-implicit local-equilibrium model with an improved parameter estimation algorithm, Industrial \& Engineering Chemistry Research 55 (2016) 7819-7829.

[31] C. S. Boeije, W. R. Rossen, Fitting foam-simulation-model parameters to data: I. coinjection of gas and liquid, SPE Reservoir Evaluation \& Engineering 18 (2015) 264-272.

[32] K. Ma, R. Farajzadeh, J. L. Lopez-Salinas, C. A. Miller, S. L. Biswal, G. J. Hirasaki, Non-uniqueness, numerical artifacts, and parameter sensitivity in simulating steady-state and transient foam flow through porous media, Transport in Porous Media 102 (2014) 325-348.

[33] L. G. Pedroni, L. Nabzar, New insights on foam rheology in porous media, Rio Oil and Gas Expo and Conference 2016 Proceedings (2016).

[34] R. Farajzadeh, M. Lotfollahi, A. A. Eftekhari, W. R. Rossen, Hirasaki, G. J. H., Effect of permeability on implicit-texture foam model parameters and the limiting capillary pressure, Energy \& Fuels 29 (2015) 3011-3018.

[35] K. Ma, J. L. Lopez-Salinas, M. C. Puerto, C. A. Miller, S. Lisa Biswal, G. J. Hirasaki, Estimation of parameters for the simulation of foam flow through porous media. part 1: The dryout effect, 2013.

[36] R. Farokhpoor, E. Westphal, N. Idowu, P. E. Oren, B. Fletcher (Eds.), gas water steady-state relative permeability determination with two approaches: experimental and digital rock analysis, strenght and weaknesses, 2016.

[37] D. K. Keelan, A practical approach to determination of imbibition gas-water relative permeability, Journal of Petroleum Technology (1976) 199-204.
[38] J. Hagoort, Fundamentals of gas reservoir engineering, volume 23 of Developments in petroleum science, Elsevier and Distributors for the U.S. and Canada, Elsevier Science Pub. Co, Amsterdam and New York and New York, NY, U.S.A., 1988.

[39] S. I. Kam, Q. P. Nguyen, Q. Li, W. R. Rossen, Dynamic simulations with an improved model for foam generation, SPE Journal 12 (2007) 35-48.

[40] A. R. Kovscek, H. J. Bertin, Foam mobility in heterogeneous porous media, Transport in Porous Media 52 (2003) 37-49.

[41] F. P. Bretherton, The motion of long bubbles in tubes, Journal of Fluid Mechanics 10 (1961) 166.

[42] Z. Zhou, W. R. Rossen, Applying fractional flow theory to foam processes at the limiting capillary pressure, SPE Advanced Technology Series 3 (1995) 154-162.

[43] L. Kapetas, S. Vincent-Bonnieu, R. Farajzadeh, A. A. Eftekhari, S. R. Mohd-Shafian, R. Z. K. Bahrim, W. R. Rossen, Effect of permeability on foam-model parameters - an integrated approach from coreflood experiments through to foam diversion calculations, $18^{\text {th }}$ European Symposium on Improved Oil Recovery, Dresden, 14-16 April (2015).

[44] R. H. Brooks, T. Corey, Properties of porous media affecting fluid flow, Journal of the Irrigation and Drainage Division IR2 (1966).

[45] W. R. Rossen, Z. H. Zhou, Modeling foam mobility at the limiting capillary pressure, SPE Advanced Technology Series 3 (1995) 146-153.

[46] H.-L. Chen, M.-J. Ke, T.-K. Chuang, R. W. Flumerfelt, Experimental studies of capillary pressure effects of foams in porous media, SPE paper 20069 (1990).

[47] V. Bergeron, C. J. Radke, Equilibrium measurements of oscillatory disjoining pressures in aqueous foam films, Langmuir 8 (1992) 3020-3026. 